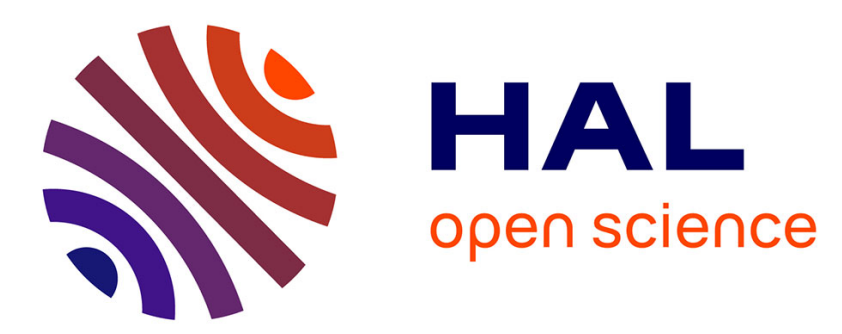

\title{
Pourquoi réformer la politique d'accueil de la petite enfance en France? Comparaison avec les politiques d'autres pays de l'OCDE.
}

Olivier Thevenon

\section{- To cite this version:}

Olivier Thevenon. Pourquoi réformer la politique d'accueil de la petite enfance en France? Comparaison avec les politiques d'autres pays de l'OCDE.. 2009. hal-00408889v2

\section{HAL Id: hal-00408889 \\ https://hal.science/hal-00408889v2}

Preprint submitted on 10 Jul 2011

HAL is a multi-disciplinary open access archive for the deposit and dissemination of scientific research documents, whether they are published or not. The documents may come from teaching and research institutions in France or abroad, or from public or private research centers.
L'archive ouverte pluridisciplinaire HAL, est destinée au dépôt et à la diffusion de documents scientifiques de niveau recherche, publiés ou non, émanant des établissements d'enseignement et de recherche français ou étrangers, des laboratoires publics ou privés. 


\title{
Pourquoi réformer la politique d'accueil de la petite enfance en France ? Comparaison avec les politiques d'autres pays de l'OCDE.
}

\author{
Olivier Thévenon ${ }^{1}$
}

Version du 09/07/2011

Mots clés : conciliation entre travail et vie familiale ; politiques familiales ; congé parental ; égalité hommes-femmes ; emploi des femmes.

Résumé : La France est souvent prise comme exemple par ses partenaires de l'OCDE pour la réussite de sa politique en faveur des familles. Son taux de fécondité élevé, combiné à un taux d'emploi des femmes supérieur à la moyenne et une proportion relativement faible d'enfants touchés par la pauvreté sont les marqueurs principaux de cette réussite. Celle-ci tient au moins en partie à l'important investissement réalisé sous formes de prestations financières, d'aides fiscales et de services d'accueil à destination de la petite enfance. Toutefois, le développement des services d'accueil ne semble pas suffisamment important face au regain du nombre de naissances observé depuis quelques années et face à la baisse de taux de scolarisation des enfants avant trois ans. De plus, l'aide accordée en cas d'interruption d'activité aux mères de deux enfants ou plus pendant trois ans est l'une des raisons d'un taux d'emploi des mères de trois enfants plus faible en France qu'en moyenne dans l'OCDE. Le système fiscal qui, contrairement à la plupart des autres pays, avantage les ménages avec un seul apporteur de revenu, ne favorise pas non plus la participation à l'emploi des mères de familles. Le manque de services d'accueil semble constituer cependant une contrainte plus déterminante. Cet article discute alors les avantages associés à une expansion des services d'accueil de la petite enfance accompagné par la mise en place d'un congé plus court mais mieux rémunéré que dans le cas présent : un bénéfice en termes de conciliation entre travail et vie familiale, d'égalité entre hommes et femmes, et de protection des familles face à la pauvreté en sont d'abord attendus. Le développement des enfants pourrait aussi en bénéficier, mais le bénéfice dépend de la qualité des services créés. L'effet sur la fécondité serait, quant à lui, vraisemblablement limité. Enfin, le « coût » d'une expansion des services d'accueil permettant de couvrir l'ensemble des enfants concernés est évalué à un montant représentant environ $0.5 \%$ du PIB, montant qui n'est sans doute pas très différent du supplément de richesse produite qui pourrait en être induit. L'investissement total pour l'accueil de la petite enfance pourrait ainsi passer de 1,3 à $1.8 \%$ de la richesse nationale.

JEL code: J08; J10; J13; J18 ; J20 ; J22

1 INED et OCDE, 2 rue André pascal, 75016 Paris : olivier.thevenon@ oecd.org. Cet article prend largement appui sur le travail publié par l'OCDE (2011), Assurer le bien-être des familles, dont Olivier Thévenon est l'un des auteurs. Willem Adema, Nabil Ali, Maria Huerta et Dominic Richardson sont vivement remerciés. Les opinions exprimées n'engagent que le présent auteur et ne peuvent en aucun cas être attribuées à l'OCDE ou ses pays membres. 
Faut-il raccourcir le congé parental en France et revoir en conséquence la politique d'accueil de la petite enfance ? Cette question, posée depuis l'introduction même du droit à un congé parental d'éducation au début des années 1980s, est remise régulièrement dans le débat public. Dernier épisode en date au mois de mai dernier : la proposition faite par l'IGAS d'étendre la durée du congé paternité, de raccourcir la durée du congé parental, et d'augmenter l'offre de garde de 200,000 places d'ici 2012 à destination des enfants de moins de trois ans. Reprenant ici une orientation déjà esquissée durant la campagne présidentielle, cette proposition est, pour autant, loin de faire l'unanimité pour plusieurs raisons. La première est qu'elle met en cause l'idéologie du «libre choix » qui porte la politique d'accueil développée en direction de la petite enfance depuis les années 1980s. Pourtant, l'argumentaire «libre-choix» est souvent dénoncé comme un «cache-sexe» d'une politique qui s'avère néfaste à l'insertion dans l'emploi des femmes dont la liberté de choix est en réalité fortement contrainte.

Une deuxième raison tient au fait qu'un raccourcissement du congé parental permettant aux parents d'assurer la prise en charge des enfants nécessite un accroissement concomitant des capacités d'accueil se substituant à la prise en charge des parents. C'est alors, l'enchaînement des modes de prise en charge de la petite enfance qui est en jeu. Or, cet enchaînement est à la croisée de différents enjeux concernant le bien-être et le développement des enfants, la préparation de leur insertion sociale et scolaire, et la conciliation entre travail et vie familiale des parents (et des femmes en particulier). Dans ce contexte, le contexte français est caractérisé par un accueil des enfants en milieu préscolaire assez précoce, mais dont le rôle est mis en cause à la fois par le dynamisme démographique et les débats récurrents sur le bien-fondé pour l'enfant d'une scolarisation précoce.

Enfin, l'investissement supplémentaire qui serait nécessaire en cas de réforme du congé parental constitue un dernier motif de résistance à une telle réforme dans un contexte où les dépenses en faveur des familles représentent déjà une proportion de la richesse nationale élevée par rapport aux autres pays de l'OCDE. Tout investissement supplémentaire ne saurait donc être envisagé sans une explicitation détaillée des bénéfices pouvant en résulter dans un contexte budgétaire très fortement contraint.

Cet article présente, en s'appuyant sur une comparaison des pays de l'OCDE, les raisons motivant une réforme des aides en matière d'accueil de la petite enfance. La première partie montre que la configuration française concilie jusqu'à présent de façon relativement satisfaisante les différents objectifs assignés aux «politiques familiales », mais que les évolutions récentes sont de nature incertaine. Les «performances » en matière de fécondité, d'emploi des femmes, ou de pauvreté des familles apparaissent comparativement élevées en moyenne, mais il existe de fortes disparités selon la situation familiale. Des conditions plus favorables à l'emploi des mères de jeunes enfants ou avec au moins deux enfants, en couple ou seules, pourrait en améliorer le taux d'emploi, et par là-même réduire les risques de pauvreté que les aides aux familles ne sont pas toujours en mesure d'éradiquer.

Dans une deuxième partie, nous rappelons certaines spécificités des transferts en faveur des familles et politiques d'accueil de la petite enfance en soulignant leurs effets contrastés sur la conciliation entre travail et prise en charge des enfants. Les justifications d'une réforme des aides en matière d'accueil sont discutées à l'aune des expériences étrangères et de la littérature analysant les interactions possibles entre l'emploi des parents, le développement des enfants et les comportements de fécondité.

La dernière partie évalue l'ordre de grandeur des dépenses additionnelles que pourrait impliquer une expansion des services d'accueil accompagnée d'un congé plus court et mieux rémunéré. Différents scénarios sont comparés pour tenir compte des choix de modes de garde qui peuvent être réalisés au cours de la petite enfance.

\section{La conciliation entre travail et vie familiale : un enjeu pour la politique d'accueil des enfants}

Les contraintes pesant sur la conciliation entre travail et vie familiale mettent en jeu plusieurs aspects des vies professionnelle et familiale : l'emploi occupé et le déroulement des carrières, la réalisation des aspirations en matière de fécondité, le bien-être et le développement des enfants, le niveau de vie général des ménages plus ou moins conforté au risque de pauvreté, ou encore l'égalité entre hommes 
et femmes dans la répartition des activités marchandes ou non. Le rôle des politiques publiques est alors d'aider les familles à concilier au mieux ces différentes dimensions et à réduire, si ce n'est éliminer les éventuels conflits qui pourraient naître entre elles. Il est alors utile, pour juger de l'opportunité d'une réforme du soutien aux parents avec de jeunes enfants, de comparer la situation de la France aux pays de même niveau de développement économique au sein de l'OCDE, sur plusieurs de ces aspects.

\subsection{Un équilibre globalement satisfaisant}

Le tableau 1 compare la situation relative des pays au regard de plusieurs indicateurs très globaux qui caractérisent les relations emploi-familles pour les années 2006 à 2009. Un constat très général peut d'abord être fait puisqu'aucun des pays ne distingue par des positions très au-dessus ou en-deça de la moyenne sur l'ensemble des indicateurs à la fois. Néanmoins, les pays nordiques et à certains égards la France combinent des «performances » relativement meilleures sur plusieurs dimensions. Ainsi, comparativement aux autres pays de l'OCDE, les «performances » françaises excèdent la moyenne sur plusieurs dimensions des vies familiale et professionnelle : la fécondité est très supérieure à la moyenne de l'OCDE et l'une des plus proche du niveau de 2,1 enfant par femme permettant le remplacement des générations ; le taux d'emploi des femmes âgées de 25 à 54 ans est à 76,6\% lui aussi supérieur à la moyenne, avec près de $80 \%$ des femmes travaillant à temps plein ; la pauvreté infantile ( $8 \%$ des enfants de 0 à 17 ans) est elle aussi très inférieure à la moyenne OCDE (12.75\%), malgré une augmentation légère ces dernières années - nous y reviendrons.

Ces résultats positifs vont de pair avec un investissement en direction des familles avec enfants $(3.8 \%$ du PIB) nettement plus élevé que la moyenne (2.2\%). L'accueil des jeunes enfant par des structures formelles y apparaît aussi nettement plus fréquent que la moyenne.

En dépit de cette situation «moyenne » très favorable, de fortes disparités existent selon la configuration familiale. D'abord, bien que le taux d'emploi des femmes en général soit comparativement élevé, celui des mères en particulier varie très fortement avec le nombre d'enfants. Les mères de un enfant continuent ainsi d'avoir un taux d'emploi (77\% en 2008) nettement supérieur à la moyenne $\mathrm{OCDE}$, mais l'écart à la moyenne se réduit pour deux enfants et s'inverse pour trois enfants ( $43 \%$ des mères de trois enfant occupant un emploi en France, contre $46 \%$ en moyenne) ${ }^{1}$. L'écart est alors très important avec la Suède, par exemple, où les trois quart des mères de trois enfants sont comptabilisées en emploi, ou avec la Finlande qui a opté pour un système de soutien aux parents qui travaillent ou qui «gardent» leur enfant de moins de trois ans plus similaire à la France. Le taux d'emploi des mères avec trois enfants est aussi inférieur en France à ce qu'il est dans des pays comme la Nouvelle-Zélande, le Canada, la Suisse ou même l'Italie et le Portugal où les taux d'emploi moyens des femmes y sont pourtant nettement inférieurs.

L'emploi des mères est aussi très significativement réduit en présence d'un très jeune enfant de moins de trois ans fortement puisque 59\% des mères françaises dans cette situation sont occupées en emploi alors qu'elles sont plus de $70 \%$ en Suède ou au Danemark. Ces mères sont aussi beaucoup plus fréquemment en emploi en Belgique, au Portugal ou aux Pays-Bas, même s'il s'agit très souvent d'un temps partiel dans ce dernier cas, parfois à faible durée hebdomadaire.

La France fait néanmoins partie des pays où les enfants de moins de trois ans sont plus fréquemment que la moyenne pris en charge par des modes d'accueil formel (42\% en France contre $32 \%$ en

\footnotetext{
${ }^{1}$ Néanmoins, une particularité forte de la France vis-à-vis des autres pays d'Europe Continentale est la forte persistance de l'emploi à temps plein (au-delà de 30 heures hebdomadaire) en présence de un enfant spécialement, voire deux enfants, les femmes se retirant plus fréquemment du marché du travail qu'elles n'optent pour un temps partiel lorsque la famille s'élargit. Le taux d'emploi à temps plein décroît ainsi fortement avec successive l'arrivée des enfants suivants : si près de $50 \%$ des mères d'un enfant travaillent à temps plein, tandis que moins de $20 \%$ sont inactives, moins de $25 \%$ des mères de trois enfants ou plus sont actives à temps plein tandis que $40 \%$ sont inactives (Thévenon, 2009).
} 
moyenne). La durée hebdomadaire de cet accueil est relativement moyenne au regard des pratiques des autres pays, cette durée étant généralement plus élevée dans les pays nordique, et plus faible dans les pays anglo-saxons (sauf aux Etats-Unis) et dans les autres pays d'Europe continentale.

\section{Tableau 1 : Indicateurs clés sur les taux de natalité, l'emploi des femmes et la couverture des} services de garde

\begin{tabular}{|c|c|c|c|c|c|c|c|c|c|c|c|}
\hline & \multirow{2}{*}{$\begin{array}{c}\text { Taux de } \\
\text { fécondité } \\
2009^{*}\end{array}$} & \multicolumn{3}{|c|}{ Taux d'emploi des femmes } & \multirow{2}{*}{$\begin{array}{c}\begin{array}{c}\text { Part du } \\
\text { travail à } \\
\text { temps partiel }\end{array} \\
\begin{array}{c}\text { femmes } 15-64 \\
\text { ans, 2009** }\end{array}\end{array}$} & \multirow{2}{*}{\begin{tabular}{|c|} 
Taux \\
d'emploi des \\
parents \\
isolés
\end{tabular}} & \multirow{2}{*}{\begin{tabular}{|c|} 
Ecart de \\
salaire entre \\
les sexes ${ }^{1}$ \\
\\
$2008^{* * *}$
\end{tabular}} & \multirow{2}{*}{\begin{tabular}{|c|} 
Pauvreté des \\
enfants $^{2}$ \\
\\
mid-late \\
$2000 s^{* * x *}$ \\
\end{tabular}} & \multirow{2}{*}{$\begin{array}{c}\text { Couverture } \\
\text { des modes } \\
\text { d'accueil } \\
\text { formels } \\
\text { (enfants }<3 \\
\text { ans) } \\
2008^{* * * * x}\end{array}$} & \multirow{2}{*}{$\begin{array}{c}\text { Durée } \\
\text { hebdomadaire } \\
\text { moyenne de } \\
\text { l'accueil }\end{array}$} & \multirow{2}{*}{$\begin{array}{c}\text { Dépenses } \\
\text { publiques en } \\
\text { faveur des } \\
\text { familles }^{3} \\
\% \text { PIB, } 2007\end{array}$} \\
\hline & & $\begin{array}{l}25-54 \text { ans, } \\
2009^{* *}\end{array}$ & \begin{tabular}{|c|} 
Mères avec \\
enfant de moins \\
de 3 ans
\end{tabular} \mid & $\begin{array}{l}\text { Mères avec } \\
\text { trois enfants }\end{array}$ & & & & & & & \\
\hline $\begin{array}{l}\text { Moyenne OCDE } \\
\text { (intervalles) }\end{array}$ & $1,74(+1-0,183)$ & $73,3(+/ .3,43)$ & $52,2(+/ .8,1)$ & $48,6(+/ .6,11)$ & $26,1(+/ .6,48)$ & $73(+/ .6,5)$ & $16(+/-4,1)$ & $12,7(+/ .3,06)$ & $32,9(+/ .8,76)$ & $27,9(+1-3,26)$ & $2,2(+1-0,46)$ \\
\hline \multicolumn{12}{|l|}{ Europe du Nord } \\
\hline Danemark & 1,84 & 82,9 & 71,4 & - & 24,8 & 83,5 & 12 & 3,7 & 72,7 & 34,0 & 3,7 \\
\hline Finlande & 1,86 & 80,4 & 51,8 & 56,1 & 15,9 & 80,0 & 21 & 4,2 & 24,2 & 34,0 & 2,7 \\
\hline Islande & 2,22 & 80,6 & - & - & 25,8 & & 13 & 8,3 & 59,7 & 36,0 & 3,5 \\
\hline Norvège & 1,98 & 83,5 & - & - & 30,4 & 72,5 & 9 & 5,5 & 44,8 & 32,0 & 2,9 \\
\hline Suède & 1,94 & 81,9 & 71,9 & 75,6 & 19,8 & 84,2 & 15 & 7,0 & 50,3 & 33,0 & 3.1 \\
\hline \multicolumn{12}{|l|}{ Europe continentale } \\
\hline Autriche & 1,39 & 79,5 & 57,9 & 48,9 & 32,2 & 73,9 & 21 & 6,2 & 12,1 & 19,0 & 3,1 \\
\hline Belgique & 1,83 & 73,8 & 65,3 & 44,6 & 31,9 & 54,7 & 10 & 10,0 & 48,4 & 29,0 & 3,1 \\
\hline France & 1,99 & 76,6 & 58,6 & 43,0 & 22,4 & 77,8 & 12 & 8,0 & 42,0 & 31,0 & 3,7 \\
\hline Allemagne & 1,36 & 75,4 & 55,5 & - & 38.1 & 56,9 & 25 & 8,3 & 27,6 & 23,0 & 2,8 \\
\hline Luxembourg & 1,59 & 71,4 & 62,5 & & 31,1 & 90,5 & - & 12,4 & 38,6 & 30,0 & 3,0 \\
\hline Pays-Bas & 1,79 & 79,6 & 75,0 & 65,4 & 59,9 & 76,2 & 17 & 9,6 & 55,9 & 19,0 & 2,9 \\
\hline \multicolumn{12}{|l|}{ Pays angloohones } \\
\hline Australie & 1,90 & 72,1 & - & - & 38,3 & 47,9 & 12 & 11,8 & 29,0 & 18,0 & 2,7 \\
\hline Canada & 1,66 & 77,2 & 58,7 & 66.3 & 27,0 & 79,8 & 20 & 14,8 & 24,0 & 32,0 & 1,4 \\
\hline Irlande & 2,07 & 67,1 & 56,1 & 43,0 & 37,4 & 54,7 & 16 & 16,3 & 30,8 & 25,0 & 2,6 \\
\hline Nouvelle Zélande & 2,14 & 74,2 & 45,1 & 56,7 & 34,5 & 64,9 & 8 & 12,2 & 37,9 & 20,0 & 2,3 \\
\hline Suisse & 1,50 & 80,6 & 58,3 & 58,0 & 46,1 & 85,7 & 20 & 9,4 & $\cdot$ & $\cdot$ & 1,4 \\
\hline Royaume-Uni & 1,94 & 74,4 & 54,0 & 41,2 & 38,8 & 47,7 & 21 & 10,1 & 40,8 & 16,0 & 3,6 \\
\hline Etats-Unis & 2.01 & 70,2 & 54,2 & & 19.2 & 80.1 & 20 & 21.6 & 31.4 & 31,0 & 1.2 \\
\hline \multicolumn{12}{|l|}{ Europe de lEst } \\
\hline République Tchèque & 1,49 & 74,1 & 17,6 & 34,6 & 6,2 & 64,4 & 21 & 10,3 & 2.2 & 16,0 & 2,4 \\
\hline Hongrie & 1,33 & 66,9 & 15,5 & 26.3 & 5,0 & 69,5 & 2 & 7,2 & 8.9 & 30,0 & 3,3 \\
\hline Pologne & 1,40 & 71,6 & 48,7 & 43,6 & 13,1 & 63,5 & 14 & 21,5 & 8,0 & 35,0 & 1,5 \\
\hline République Slovaque & 1,41 & 71,2 & 21,6 & 36,3 & 4,1 & - & - & 10,9 & 3,0 & 29,0 & 2,2 \\
\hline \multicolumn{12}{|l|}{ Europe du Sud et Asie } \\
\hline Grèce & 1,53 & 62,2 & 50,9 & 40.1 & 14,4 & 86,5 & 10 & 13,2 & 15,7 & 30,0 & 1,3 \\
\hline Italie & 1,41 & 59,1 & 51,1 & 51,4 & 30,5 & 86,5 & 1 & 15,3 & 29,2 & 29,0 & 1,4 \\
\hline Portugal & 1,32 & 74,9 & 67,6 & 51,4 & 13,8 & 88,7 & 16 & 16,6 & 47,4 & 38,0 & 1,3 \\
\hline Espagne & 1,40 & 63,8 & 54,8 & 40,8 & 21,4 & 81,8 & 12 & 17,3 & 37,5 & 28,0 & 1,6 \\
\hline Japon & 1,37 & 67,6 & 29.8 & - & 33,8 & 86,0 & 31 & 14,2 & 28,3 & - & 1,3 \\
\hline Corée & 1,15 & 59.8 & - & - & 14,2 & 69,0 & 39 & 10,3 & 37,7 & - & 0,6 \\
\hline \multicolumn{2}{|c|}{ Supérieur à la moyenne OCDE } & & & & \multicolumn{2}{|c|}{\begin{tabular}{|c|}
$\begin{array}{c}\text { Au niveau de la moyenne OCDE } \\
\text { (ou donnée non disponible) }\end{array}$ \\
\end{tabular}} & & & & \multicolumn{2}{|c|}{ Inférieur à la moyenne OCDE } \\
\hline
\end{tabular}

Les pays sont classés dans les catégories "supérieur" ou "inférieurs » à la moyenne 'OCDE" s'ils se situent à un demi écart-type au-dessus ou au-dessous de la moyenne de l'OCDE. Le seuil d'un demi écart-type fournit une approximation de l'intervalle de confiance à $99 \%$ estimé dans un cas d'échantillon compris entre 25 à 30 pays selon les variables. Autrement dit, on repère ici les pays qui s'écartent de la moyenne avec seulement 1 chance sur 100 de se tromper.

(1) L'écart de salaire n'est pas ajusté et renvoie à la différence de revenus du travail médians entre hommes et femmes (i.e. revenus médians des femmes relativement à celui des hommes). Le calcul est basé sur les revenus bruts.

(2) Le taux de pauvreté des enfants est la proportion d'enfants vivant dans un ménage percevant moins de la moitié du revenu par unité de consommation médian. Les données proviennent du questionnaire OCDE sur la distribution des revenus, février 2011. (www.oecd.org/els/social/inequality).

(3) Les dépenses publiques en faveur des familles incluent les prestations perçues uniquement par les familles avec enfants (allocations et prestations familiales, prestations versées en cas de congé maternité ou parental, et aides à prise en charge des enfants de moins de 6 ans). Les suppléments accordés aux familles en matière de santé ou de logement ne sont pas inclus ici. Les données sur les avantages fiscaux bénéficiant aux familles ne sont pas disponibles pour la Grèce, l'Italie, l'Estonie, la Slovénie, la Finlande, le Luxembourg, la Hongrie, le Danemark et l'Islande.

* 2007 pour le Canada et les Etats Unis

** 2006 pour la Suisse; 2005 pour l'Australie, le Japon, la Nouvelle Zélande et les Etats unis; 2002: Islande; 2001: Canada; 1999: Danemark

*** 2006 pour l'Australie. Données concernant l'ensemble des parents isolés pour le Canada, Danemark, Islande, Nouvelle Zélande, Norvège et Suède. Ces taux sont généralement légèrement plus élevés que dans le cas des mères seules.

**** 2009 pour le Mexique ; 2008 pour la Nouvelle Zélande et la Corée ; 2005 pour l'Australie et les Etats Unis. Données issues de EU SILC 2006 pour les pays européens. Les taux d'accueil incluent les différents modes de garde formel comme les centres collectifs ou les gardes à domicile par des assistantes maternelles agréées.

${ }_{\star \star * * \star}$ Le taux d'équivalent temps plein mesure la proportion d'enfants gardés pour un équivalent égal à 30 heures par semaine. II est obtenu par le produit suivant : Proportion d'enfants accueillis formellement*nombre moyens d'heures hebdomadaire (en\% de 30 heures par semaines)

${ }_{* * * * *}$ Le taux de pauvreté infantile est défini comme la part des enfants vivant dans des ménages dont le revenu par unité de consommation est inférieur à $50 \%$ du revenu médian de toute la population.

***** L'écart de salaire est mesuré ici en référence au revenu médian des employées à temps plein. Données issues de la Base OCDE sur les revenus.

Source : Base OCDE sur la famille. 


\section{2...mais fragilisé par les évolutions démographiques récentes}

Certaines évolutions en cours fragilisent néanmoins l'équilibre atteint. D'abord les taux de fécondité connaissent une croissance persistante depuis quelques années après plusieurs décennies de baisse assez marquée. Cette remontée observable depuis la fin des années 1990s indique que les générations les plus jeunes ont leurs enfants à des âges plus avancés que leurs aînés, avec une forte croissance des naissances à un âge de la mère supérieur à 30 ans (Prioux, 2009). Cet accroissement du nombre de naissances a lieu dans un contexte où le nombre de places offertes à l'accueil de la petite enfance augmente certes, mais moins rapidement que le nombre de naissances. Ainsi de 1998 à 2006, 47,000 places supplémentaires en services d'accueil ont été créées, alors que le nombre de naissances s'est accru annuellement de 61,000.

La croissance du nombre de familles monoparentales est un facteur qui favorise cette extension de la pauvreté. Ainsi, l'OCDE note que la proportion de personnes vivant dans une famille monoparentale est passée de $7.3 \%$ en France au mitan des années 1980s à 12.7\% au milieu des années 2000s (ces taux étant supérieurs aux proportions respectivement de $5.5 \%$ et $7.7 \%$ en moyenne dans l'OCDE). Le taux de pauvreté de ces familles apparaît relativement stable au cours des deux dernières décennies (autour de $20 \%$, contre $31 \%$ en moyenne dans l'OCDE) ${ }^{2}$, mais leur risque de pauvreté est beaucoup plus fort que celui encouru par d'autres situation familiale, y compris et surtout les couples avec enfants. Pour l'INSEE, le risque de pauvreté des personnes vivant au sein d'une famille monoparentale est 2,3 fois plus important que l'ensemble de la population. A l'inverse, ce sont les couples sans enfant qui sont les moins touchés par la pauvreté (Godefroy et al. 2011). Au total, ce sont environ $8 \%$ des enfants de moins de 18 ans qui sont touchés par la pauvreté en France (contre 12,7\% en moyenne dans l'OCDE), mais leur risque d'être dans cette situation dépend des situations à la fois conjugales et professionnels de leur parents.

Ainsi, parmi les enfants vivant au sein de familles monoparentales, 35,8\% d'entre eux vivent dans un ménage pauvre lorsque le parent n'a pas d'emploi, tandis que ce risque est réduit ) $14.6 \%$ lorsque ce parent a un emploi. La forte réduction du risque de pauvreté induite par l'emploi des parents est aussi visible dans les couples avec enfants. Ainsi, $18 \%$ des enfants vivant avec deux parents sont touché par la pauvreté lorsqu'aucun de ces parents n'occupe d'emploi ; mais ce taux passe à $9 \%$ lorsqu'un seul des parents a un emploi et seulement 3\% lorsque les deux parents ont un emploi (OCDE, 2011).

Au total, l'équilibre atteint en France apparaît plutôt satisfaisant au regard de la moyenne des pays rassemblés ici. Néanmoins, l'écart avec les pays les plus «performants » demeure significatif sur plusieurs points. En particulier, les taux d'emploi des femmes ayant des enfants à charge sont nettement plus élevés dans la plupart des pays nordiques, spécialement pour les mères de très jeunes enfant ou de famille nombreuse, ou les parents isolés. Il en résulte aussi un taux de pauvreté qui affecte plus fréquemment les enfants en France, même si là encore cette dernière est dans une situation nettement plus favorable que la moyenne. Les évolutions plus ou moins récentes de nature démographique (regain de la fécondité, croissance du nombre de familles monoparentales). Ces évolutions démographiques ont lieu dans un contexte de croissance limitée des services d'accueil ouverts aux très jeunes enfants. L'équilibre atteint, s'il est donc plutôt bien meilleur qu'en moyenne, apparaît fragilisé par ces évolutions. Cette fragilisation pourrait atteindre le cœur même de ce qui fait la spécificité française en matière de politique en faveur des familles avec jeunes enfants. Cette spécificité est décrite dans la section qui suit.

\footnotetext{
${ }^{2}$ L'OCDE retient une définition du seuil de pauvreté fixé à la moitié du revenu médian. Le taux de pauvreté est alors inférieur à celui obtenu lorsque ce seuil correspond à $60 \%$ du revenu médian. Le taux de pauvreté des familles monoparentale est alors d'environ 30\%, selon l'INSEE (Godefroy et al., 2011).
} 


\section{La spécificité française en matière d'accueil de la petite enfance}

\subsection{Une aide publique aux familles relativement ample et diversifiée en France}

Comme nous l'avons déjà souligné, la France fait partie des pays où le montant des ressources publiques investies en direction des familles avec enfants est le plus élevé en proportion de la richesse nationale. D'autres spécificités caractérise la répartition ces dépenses, à commencer par la part relativement importante jouée par les avantages fiscaux dont bénéficient ces familles, dont une partie au titre d'aides à la garde des enfants (graphique 1).

\section{Graphique 1: La France se positionne en tête des pays de l'OCDE en matière de dépenses en faveur des familles}

Panel A : Dépenses publiques en faveur des familles en prestations financières, services d'accueil et avantages fiscaux, en pourcentage du PIB, 2005

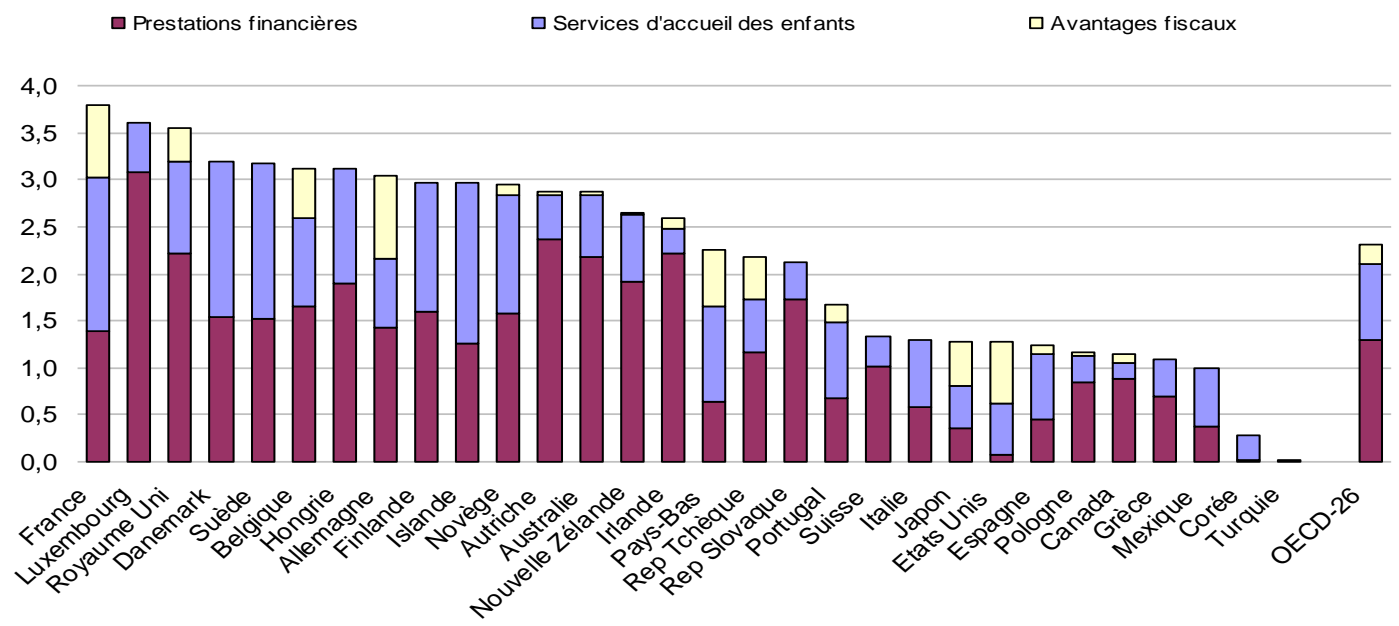

Panel B: Répartition des dépenses en services pour les enfants de moins de 6 ans

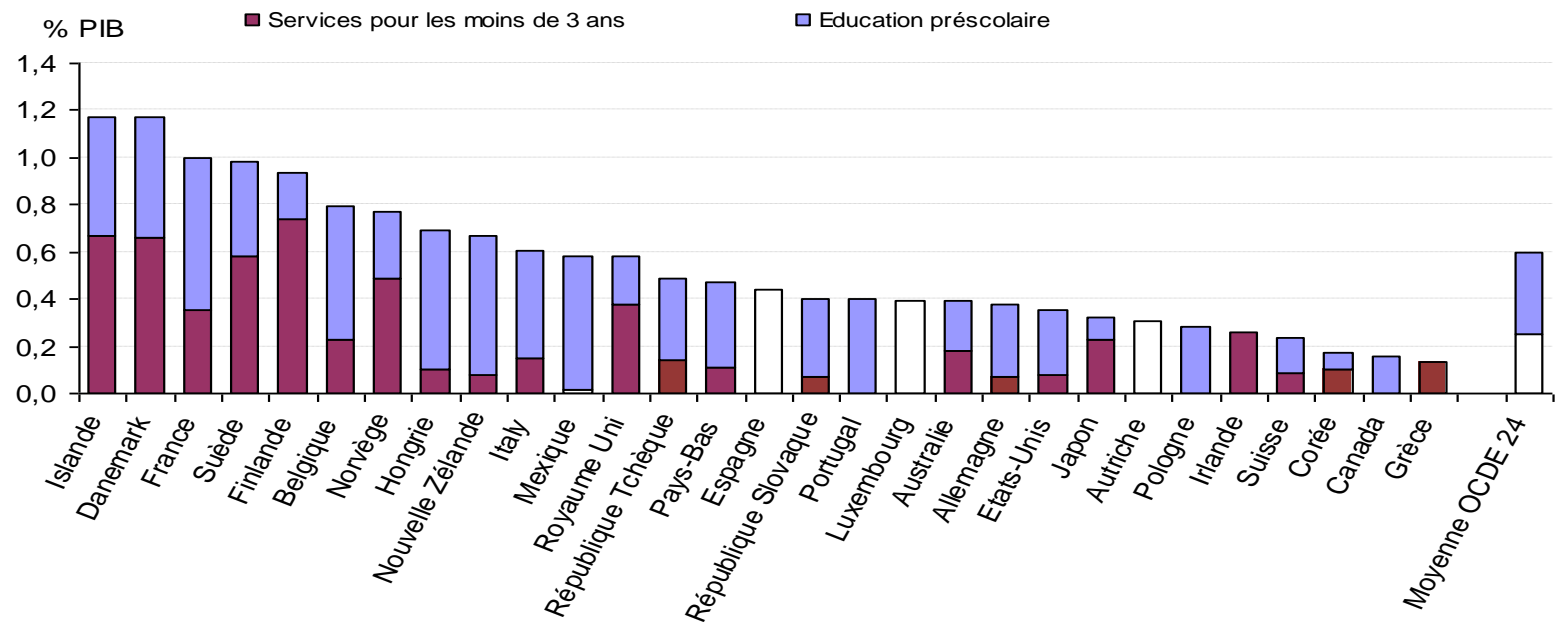

Les aides publiques concernent ici les aides exclusivement versées aux familles (ex. prestations et allocations pour enfants, revenu perçu durant les congés parentaux et les aides en matière de garde). Les dépenses enregistrées dans les autres secteurs de l'aide sociale comme la santé ou les aides en matière de logement ou d'assistance sociale ne sont pas incluses. Les dépenses en "services " n'incluent ici que les dépenses d'accueil pour la petite enfance avant l'entrée en école primaire : subventions aux prestataires de services de garde et d'éducation préscolaire. Les aides fiscales octroyées aux parents en matière de garde sont incluses parmi les avantages fiscaux avec les autres formes d'aides fiscales.

Les dépenses en « services d'accueil » incluent ici uniquement les dépenses d'accueil pour la petite enfance avant l'entrée en école primaire : les subventions aux prestataires de services de garde couvrent généralement les enfants de moins de trois ans, tandis que les services d'éducation préscolaire couvrent les enfants de 3 ans à l'âge d'entrée en école primaire qui varie entre 6 et 7 ans selon les pays.

Source: Base de données sur la famille (www.oecd.org/els/social/family/database). 
La part importante des dépenses réalisées en matière de services d'accueil est aussi un caractère que partage la France avec les pays nordiques - à l'inverse par exemple de pays comme l'Allemagne où cet investissement demeure limité au profit d'aides financières versées aux familles. Dans ces dépenses en services, la part réservé à l'éducation préscolaire est très fortement dominante, alors que les dépenses réalisées pour l'accueil des enfants de moins de trois ans est sensiblement plus important dans les pays nordiques (graphique 2, Panel 2B). Les dépenses en éducation préscolaire représentent ainsi $0,64 \%$ du PIB en France, contre 0.3 en moyenne dans l'OCDE (et $0.4 \%$ dans les pays nordiques). Inversement, $0.4 \%$ du PIB est investi dans les modes d'accueil des enfants de moins de trois ans en France, ce qui est supérieur à la moyenne OCDE (0.2\%), mais nettement inférieur aux $0.6 \%$ dépensés par les pays nordiques. La plus grande couverture de ces services au sein de la population des enfants de moins de trois ans, et la durée hebdomadaire moyenne relativement élevée pendant laquelle ces enfants sont accueillis dans ces services expliquent en partie les plus grandes dépenses qui sont faites dans cette direction dans ces pays (avec de fortes variations entre ces pays néanmoins).

\subsection{Des taux effectif d'imposition comparativement modérés}

Pour comprendre la façon dont les résultats du tableau 1 sont liés entre eux, l'impact des transferts sur le niveau de vie des familles peut être regardé selon deux angles. On compare, d'une part, l'effet du système socio-fiscal sur le niveau global de prélèvement qui est réalisé dès lors qu'un parent entre activité. On examine, d'autre part, la façon dont, en l'absence d'emploi, les transferts maintiennent ou non les familles à des niveaux de vie supérieurs aux seuils de pauvreté.

L'examen de l'impact des transferts financiers sur le niveau de familles montre que l'entrée dans l'emploi d'un deuxième actif au sein d'un ménage - ici avec deux enfants - est imposée à un niveau relativement limité en comparaison des niveaux d'imposition effectifs des autres pays de l'OCDE. Le graphique mesure, pour observer cela, l'augmentation du prélèvement effectif (tenant compte de l'ensemble des transferts socio-fiscaux qui s'ajoutent ou se déduisent) réalisé sur les revenus induits par une entrée dans l'emploi d'un second actif dans les couples (graphique 2, Panel A) ou d'un parent en situation de monoparentalité. Ce taux est ainsi mesuré en pourcentage du revenu brut additionnel perçu par le ménage. En France, environ $46 \%$ du revenu brut perçu par un deuxième emploi rémunéré au salaire moyen dans un couple marié ainsi prélevé sous forme d'impôts ou de cotisations - lorsque le conjoint est supposé lui aussi percevoir un salaire de niveau moyen. La France se situe alors dans la moitié de pays où ce taux est inférieur à la moyenne.

Les parents en situation de monoparentalité font, quant à eux, face à une hausse du taux effectif d'imposition induite par une transition vers l'emploi très proche de la moyenne OCDE, en raison d'une baisse marquée des prestations familiales et sociales lors du passage à l'emploi. Le pourcentage de revenu prélevé de façon nette est ainsi plus élevé pour un parent seul $(65 \%)$ que pour un parent dont le conjoint perçoit aussi un revenu égal au salaire moyen (46\%), et il est même de $78 \%$ lorsque le revenu issu du nouvel emploi est égal à la moitié du salaire moyen (Panel B). Les taux de prélèvements demeurent néanmoins en France inférieurs à la moyenne. 
Graphique 2 : Taux d'imposition effectif du revenu acquis par une entrée en emploi - 2008.

Panel A : Taux d'imposition effectif d'un couple avec deux enfants ${ }^{l}$

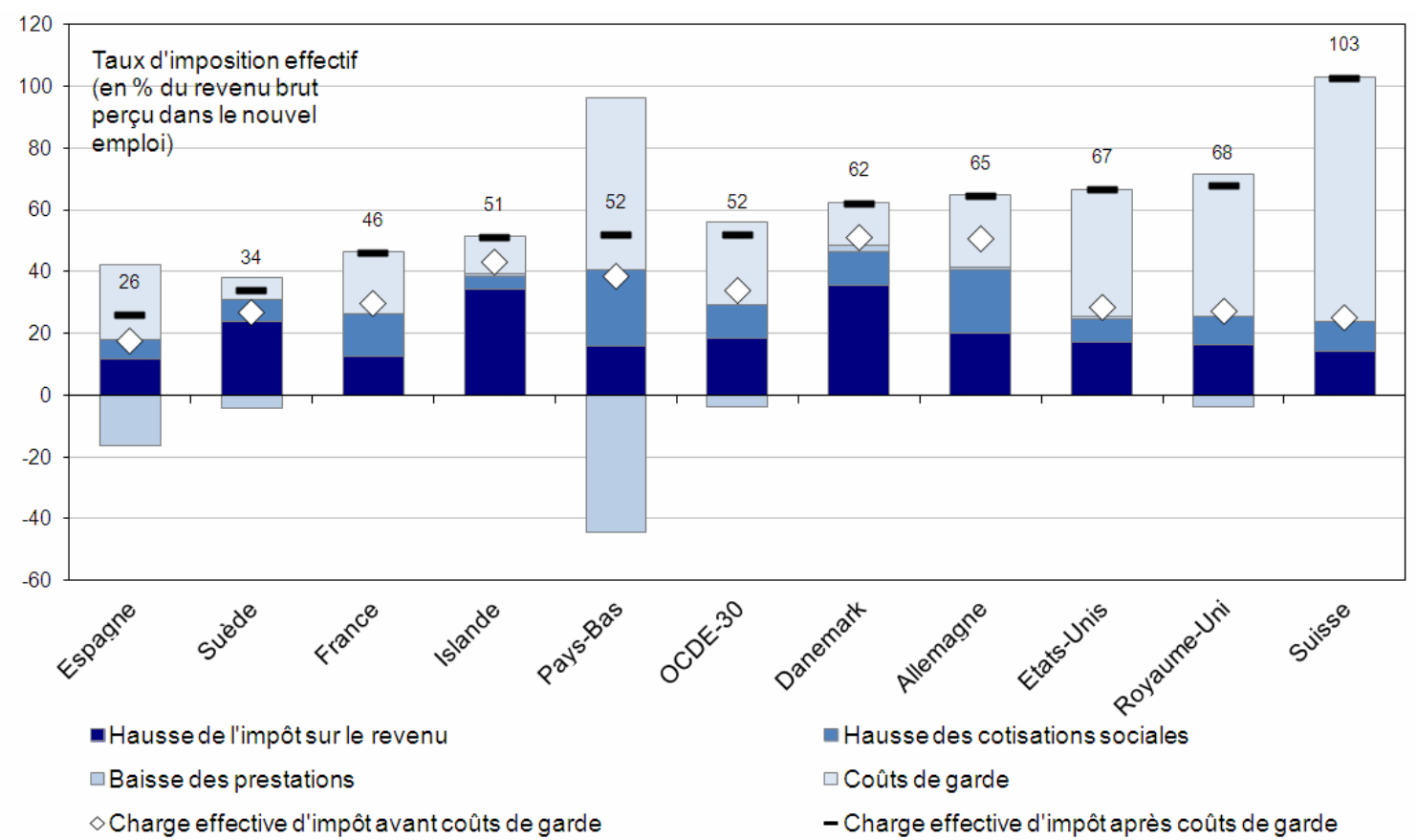

Panel B : Taux d'imposition effectif du revenu acquis par un parent seul avec deux enfants ${ }^{l}$

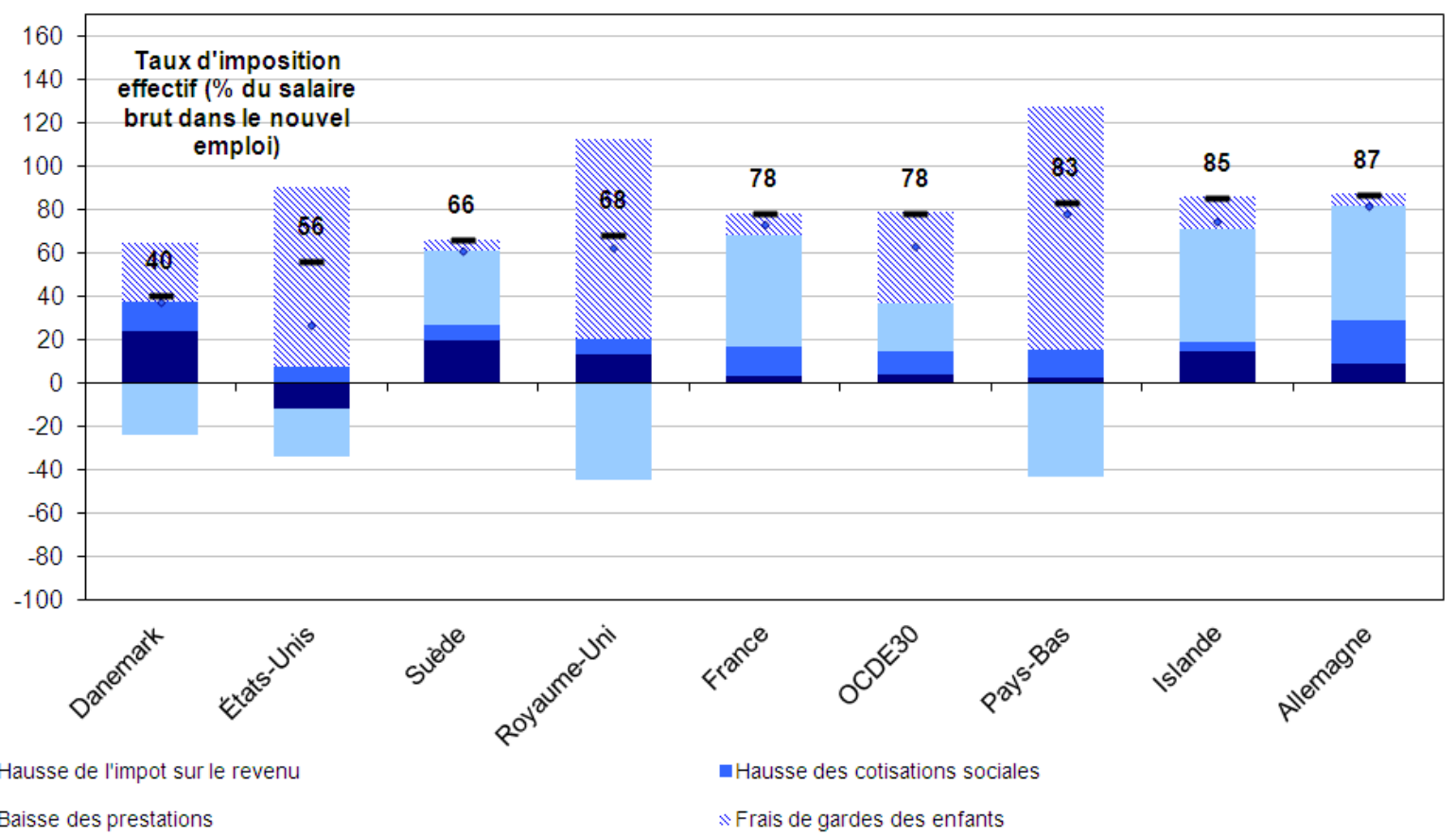

- Hausse total de la charge fiscale effective sans frais de garde des enfants - Hausse total de la charge fiscale effective avec frais de garde des enfants

1) hypothèses : panel $A$ concerne les couples mariés; passage d'une situation initiale avec un seul actif dans le ménage à deux actifs, chacun percevant un salaire égal au niveau moyen. Panel B : le parent "seul » est supposé entrer dans un emploi rémunéré à $50 \%$ du salaire moyen. Dans les deux cas, deux enfants sont supposés présents dans le ménage, de deux et trois ans accueillis en crèche. 
Toutefois, la France fait partie des rares pays où les couples (mariés) composé d'un seul apporteur de revenus sont imposés de façon plus favorables que ceux où, à revenu total identique, deux apporteurs de revenus y contribuent. Le Tableau 2 illustre pour quelques pays la différence de taux d'imposition effectif s'appliquant à des couples mariés avec deux enfants de 6 et 11 ans dont la distribution du revenu varie au sein des ménages. La dernière colonne du tableau mesure l'avantage fiscal bénéficiant aux couples mono-actifs par différence d'imposition nette entre les ménages à un seul ou deux revenus. Le solde est négatif en France, comme en Allemagne mais avec une différence de taux d'imposition moins forte. Cette différence n'est pas très forte mais elle contraste nettement avec les soldes largement positifs observés dans les autres pays.

Tableau 2 : Taux d'imposition des couples mariés en fonction de la répartition du revenu entre les conjoints

Taux d'imposition effectifs en \% du revenu total brut du ménage, pour des couples mariés avec deux enfants de 6 et 11 ans, dont le revenu total est égal à 133\% ou 200\% le revenu moyen, 2008

\begin{tabular}{l|cc|cc|cc|cc} 
& $\begin{array}{c}\text { Couple à un seul } \\
\text { actif }\end{array}$ & $\begin{array}{c}\text { Couple avec un } \\
\text { apporteur de } \\
\text { revenus } \\
\text { dominant }\end{array}$ & $\begin{array}{c}\text { Deux apporteurs } \\
\text { de revenus } \\
\text { égaux }\end{array}$ & $\begin{array}{l}\text { Différence d'imposition } \\
\text { nette entre les ménages } \\
\text { à un seul et deux } \\
\text { apporteurs de revenus }\end{array}$ \\
$\begin{array}{l}\text { Taux d'imposition (\% du } \\
\text { revenu brut, avant coûts } \\
\text { de garde) }\end{array}$ & $133-0$ & $200-0$ & $100-33$ & $150-50$ & $67-67$ & $100-100$ & 133 & 200 \\
& $\mathbf{1}$ & $\mathbf{2}$ & $\mathbf{3}$ & $\mathbf{4}$ & $\mathbf{5}$ & $\mathbf{6}$ & $\mathbf{7}$ & $\mathbf{8}$ \\
\hline Danemark & 35,9 & 43,9 & 33,5 & 41,0 & 33,4 & 37,5 & 7,0 & 14,7 \\
\hline France & 19,7 & 23,0 & 17,9 & 23,3 & 20,0 & 23,7 & $-1,7$ & $-2,9$ \\
\hline Allemagne & 27,5 & 30,4 & 28,9 & 34,7 & 28,9 & 36,7 & $-5,2$ & $-20,6$ \\
Islande & 13,6 & 22,1 & 13,7 & 22,2 & 13,7 & 22,2 & $-1,1$ & $-0,4$ \\
Italie & 25,0 & 34,2 & 18,2 & 27,4 & 18,2 & 25,8 & 27,0 & 24,8 \\
Pays-Bas & 28,4 & 35,7 & 23,1 & 29,8 & 22,4 & 29,3 & 21,2 & 17,9 \\
Suède & 27,4 & 36,8 & 18,9 & 28,2 & 18,3 & 23,2 & 33,3 & 37,1 \\
Royaume-Uni & 22,8 & 29,7 & 18,1 & 24,5 & 18,1 & 23,2 & 20,8 & 21,9 \\
Etats-Unis & 12,0 & 17,7 & 12,0 & 17,7 & 12,0 & 17,7 & 0,0 & 0,0 \\
OCDE & 20,5 & 27,5 & 16,8 & 23,9 & 16,0 & 23,0 & 22,7 & 15,7 \\
\hline
\end{tabular}

Source : OECD modèle de simulation des prestations et impôts; indicateur PF4.1 Base de données sur la Famille.

\subsection{Un risque de pauvreté persistent en cas de non-emploi}

Un autre aspect des transferts réalisés en France en direction des familles avec enfants, est qu'ils apparaissent comparativement efficaces pour préserver les familles de la pauvreté extrême en octroyant des prestations universelles ou ciblées aux plus démunis (OCDE, 20XX). Ces prestations ne parviennent toutefois pas toujours à tenir les familles très à l'écart de la pauvreté, notamment lorsque la naissance d'un enfant entraîne une interruption d'activité de la mère. Le graphique 3 illustre pour cela l'évolution simulée du revenu après transferts en fonction de l'âge de l'enfant de manière à suivre l'effet de la naissance et du revenu de remplacement obtenu lorsque le ou l'un des parents interrompt son activité pour prendre un congé parental (à l'issue du congé maternité). Ce revenu est simulé pour trois cas de ménage à revenus relativement faibles, c'est-à-dire dont le revenu total est égal au salaire national moyen ou à sa moitié. On voit alors que le recours au congé parental qui suit la naissance d'un enfant induit une baisse de revenu relativement forte en France, particulièrement pour les familles (ici avec 4 enfants ou plus) et les familles monoparentales. Par comparaison, l'incidence de la naissance sur le niveau de vie des ménages est nettement moindre en Allemagne ou au Danemark, en raison de la rémunération perçue par le parent en congé parental, supérieure à la France. Les ménages avec de faibles revenus du travail (50\% du salaire médian) sont en outre tenus plus nettement à l'écart de la pauvreté dans ces deux pays. 


\section{Graphique 3 : Effets des transferts socio-fiscaux sur le risque de pauvreté des familles}

\section{Ratios revenu familial net disponible par type de famille / seuil de pauvreté pour la population totale}

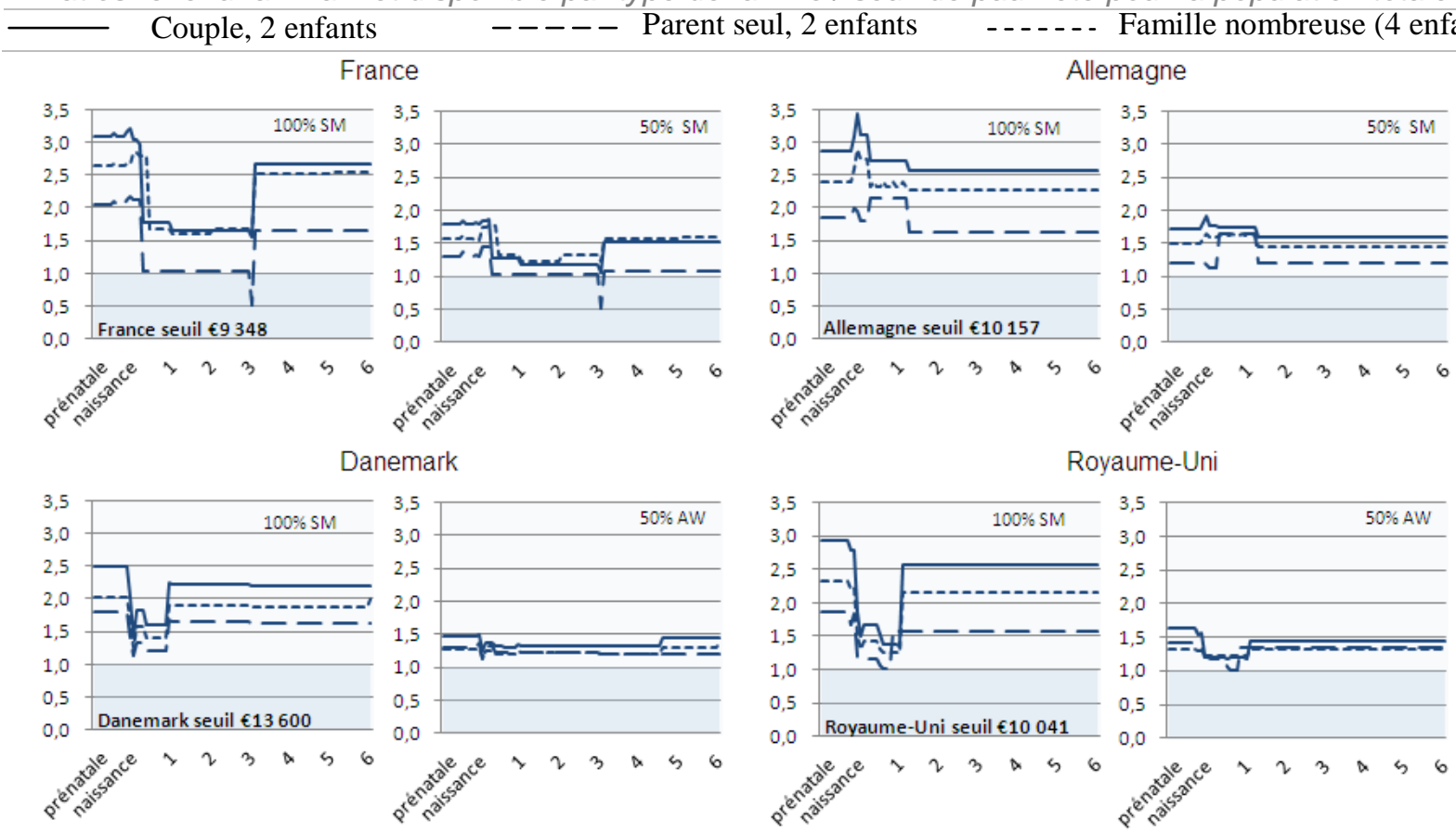

1) L'ordonnée de chaque graphique est normalisée au seuil de pauvreté égal à 1 . Ce seuil de pauvreté relative est fixé à 50 \% du revenu médian équivalent des ménages. Les graphiques de la partie gauche représentent les variations de revenus dans les premières années pour les familles gagnant $100 \%$ du salaire moyen national (les parents travaillent toujours à temps plein excepté pendant les périodes de congé) ; les graphiques de la partie droite correspondent aux familles gagnant $50 \%$ salaire moyen national.

${ }^{1}$ Les familles biparentales ont deux apporteurs de revenus travaillant à temps plein ; les familles monoparentales ont un apporteur de revenus travaillant à temps plein. Les zones grisées représentent les revenus inférieurs au seuil national de pauvreté relative. Les valeurs portées sur l'axe des y sont les valeurs de ratio relatives au seuil de pauvreté. On suppose dans le modèle que les parents prennent tous les congés auxquels ils peuvent prétendre, quel que soit le niveau de paiement.

Source: OCDE (2011), Assurer le bien-être des familles, OCDE Publications.

Dans ce contexte, l'emploi des parents - y compris de ceux étant en situation de monoparentalité constitue une protection forte et souvent indispensable pour les familles face à la pauvreté. Pour autant le taux d'emploi des parents «isolés» paraît relativement moyen en France (78\%), comparativement à ceux observés globalement dans les pays du Nord et du Sud de l'Europe. Ces parents sont dans une très grande majorité des mères qui font face plus souvent face à des contraintes de modes de garde que pour les autres types de famille (Eydoux et Letablier, 2007). De plus, ces parents ne sont pas moins exposés aux horaires atypiques que les autres parents, et ils occupent plus souvent un emploi à faible salaire (notamment les pères comparés à ceux vivant en couple) (Eydoux et Letablier, 2007). Les mères isolées ont alors recours à des solutions de garde « collectives », moins onéreuses que les solutions individuelles comme, par exemple, les assistantes maternelles, mais dont les horaires sont moins souples (Eydoux et al. 2006). Elles recourent aussi plus souvent à des aides informelles et combinent plusieurs modes de garde.

\subsection{Une aide ambivalente pour les familles avec un enfant de moins de trois ans}

Plusieurs caractéristiques de la politique d'accueil de la petite enfance justifient cette répartition des dépenses. La politique de «choix» d'accueil ainsi développée en France présente plusieurs spécificités dont la combinaison distingue le système français des autres pays de l'OCDE (Thévenon, 2006 ; Adema et Thévenon, 2008). La France apparaît ainsi sans doute comme le pays allant le plus loin dans la diversification des modes d'accueil des petits enfants, et des aides publiques soutenant chacun de ces modes. Ainsi, une politique de «double» libre-choix a été progressivement mise en place, qui signifie que les parents doivent avoir le choix (si ce n'est en pratique en théorie), d'une part, entre la possibilité de s'occuper eux-mêmes de leur très jeune enfant ou d'utiliser un mode d'accueil 
extrafamilial ; un autre choix s'effectue, d'autre part, dans ce dernier cas entre des modes d'accueil très divers, collectifs (crèches, halte-garderies, jardins d'enfants), familiaux (crèches parentales) ou individuels (assistantes maternels), le recours à chacun de ces modes étant subventionné.

Cette politique de «libre-choix » se décline avec plusieurs très particuliers. D'abord, les parents qui interrompent partiellement ou complètement leur activité pour s'occuper de leur enfant dans les mois ou années suivant la naissance peuvent bénéficier d'une allocation (pour une période qui varie avec le rang de naissance: six mois pour le premier enfant, jusqu'au troisième anniversaire de l'enfant à partir du deuxième. Le montant de cette allocation est forfaitaire, égal à $536 €$ en 2008 soit environ un demi SMIC. Son bénéfice peut être associé au recours par l'un des parents à un congé parental. Ce type d'allocation existe dans plusieurs autres pays européens (par exemple en Autriche, Finlande, Hongrie, Norvège, etc.), la variation de la durée de versement en fonction du rang de naissance est ici spécifique. Elle résulte, fondamentalement, du souhait de ne pas créer de trop fortes incitations à une interruption longue en début de carrière qui se conjugue très souvent avec l'arrivée du premier enfant auprès de femmes qui connaissent des difficultés d'insertion sur le marché du travail. Est le résultat spécifique d'un compromis entre l'objectif de permettre aux parents qui le souhaitent de prendre en charge leur jeune enfant, et celui de promouvoir l'emploi des femmes et sa conciliation avec la vie familiale (Encadré 1).

Par ailleurs, l'accès précoce à des structures d'accueil collectif est une caractéristique forte que partage pour une part la France avec les pays nordiques, mais selon certaines modalités spécifiques. Tout d'abord, l'accueil en crèche peut débuter dès l'âge de 3 mois en France, alors que les enfants ne sont accueillis dans ces structures qu'à partir de l'âge de 1 an ou 18 mois dans les pays nordiques. De plus, ces structures offre un service quotidien suffisamment étendu pour être souvent compatible avec l'emploi à temps plein des parents, comme dans les pays nordique, mais à la différence de pays comme l'Allemagne, les Pays-Bas ou le Royaume-Uni où l'offre d'accueil est très souvent limitée à un temps partiel.

L'entrée en école maternelle peut aussi s'effectuer de façon plus précoce en France que la beaucoup d'autres pays, puisqu'elle peut avoir lieu avant trois ans bien que les communes n'aient aucune obligation stricte en ce sens. Néanmoins, l'accès à l'école maternelle à l'âge de deux ans qui en concernait plus d'un tiers (35\%) en 2000 a fortement baissé puisque seuls $13.6 \%$ des enfants de deux ans fréquentent un établissement préscolaire en 2010. La baisse du nombre de places mises à disposition de cette population en est la principale cause puisque que ce nombre est passé de 254,000 en 2001 à 123,300 en 2009.

Enfin, les ménages plus aisés et souhaitant préserver un contexte familial ou un mode de garde plus souple peuvent assistantes maternelles qui bénéficient d'aides sous la formes de réduction d'impôts liées aux frais de garde et/ou à l'emploi de services à domicile. 


\section{Encadré 1 : Le congé parental en France et sa rémunération}

En France, tout parent salarié satisfaisant les conditions d'éligibilité a droit à un congé parental d'au plus trois années après la naissance d'un enfant, à l'issue duquel le salarié retrouve son emploi ou un emploi similaire. Ce droit individuel peut être combiné au bénéfice d'une allocation versé au ménage en cas de réduction des heures de travail ou d'interruption d'activité. Les conditions entourant cette allocation ont fortement évolué au cours du temps.

L'Allocation Parentale d'Education fut d'abord introduite en 1985 pour les parents décidant d'interrompre leur activité ou de réduire leur durée du travail à la naissance d'un troisième enfant. L'allocation peut alors être perçue pour une période maximale de 36 mois. Le droit à cette allocation fut ensuite étendu à la naissance d'un deuxième enfant en 1994. Les femmes forment la très grande majorité des bénéficiaires de cette allocation et d'un congé parental.

Les parents donnant naissance à un premier enfant étaient donc exclus du dispositif, ceci résultant d'un compromis entre deux argumentaires (Thévenon, 2006). D'un côté, le soutien à la garde parentale était clairement promu au même titre que l'accueil extra-familial, et se trouvait confortée par des considérations de politiques d'emploi percevant l'octroi de cette allocation comme un moyen de limiter la pression exercée sur le marché du travail par l'offre croissante de travail des femmes ; d'un autre côté, l'exclusion du premier enfant était porté, outre par des considérations budgétaires, par la volonté de ne pas porter préjudice à la carrière des femmes qui s'établissent généralement sur le marché du travail en même temps qu'elles ont leur premier enfant.

La réforme de la Prestation d'Accueil du Jeune Enfant (PAJE) adoptée en 2004 étant toutefois le dispositif au premier enfant, mais pour une période plus courte et avec des conditions d'éligibilité spécifiques. Le Complément de Libre Choix d'Activité (CLCA) donne ainsi droit à une allocation perçue pendant une période maximale de 6 mois.

Par ailleurs, les parents d'une troisième enfant peuvent aussi opter pour le Complément Optionnel de Libre Choix d'Activité (COLCA) qui offre une allocation d'un montant supérieur ( $€ 767$ en 2008 contre $€ 536$ pour le CLCA) mais pour douze mois. Le succès de ce dispositif est néanmoins très limité puisque on dénombrait 2600 bénéficiaires à taux plein en 2008, du COLCA, soit à peine $1,7 \%$ des bénéficiaires d'un complément de libre choix.

La France fait partie des pays où les parents de très jeunes enfants peuvent bénéficier d'une période de congé parental relativement longue - près de 36 mois au total - et bénéficier durant cette période d'une allocation fixe et d'un montant limité. Au total, le taux de remplacement global apparaît assez limité pour un salarié qui recevra au terme de ces 36 mois de congé l'équivalent de 31 semaines rémunéré au taux de salaire moyen. In fine, ce taux de remplacement global n'est pas très différent de celui existant non seulement dans d'autres pays doté de droits assez similaires, mais aussi au Danemark ou en Islande où un congé est accordé pour une période nettement plus courte et le mode de paiement lié au salaire (Encadré 2). Rapportées au nombre de naissances, les dépenses réalisées au titre des congés maternité et parental demeurent relativement faibles en France comparées aux pays nordiques et quelques pays de l'Est européen. 


\section{Graphique 4 : Caractéristiques des congés maternités et parentaux, 2008}

Panel A : Durée en semaines du congé maternité, et équivalent taux plein ${ }^{l}$

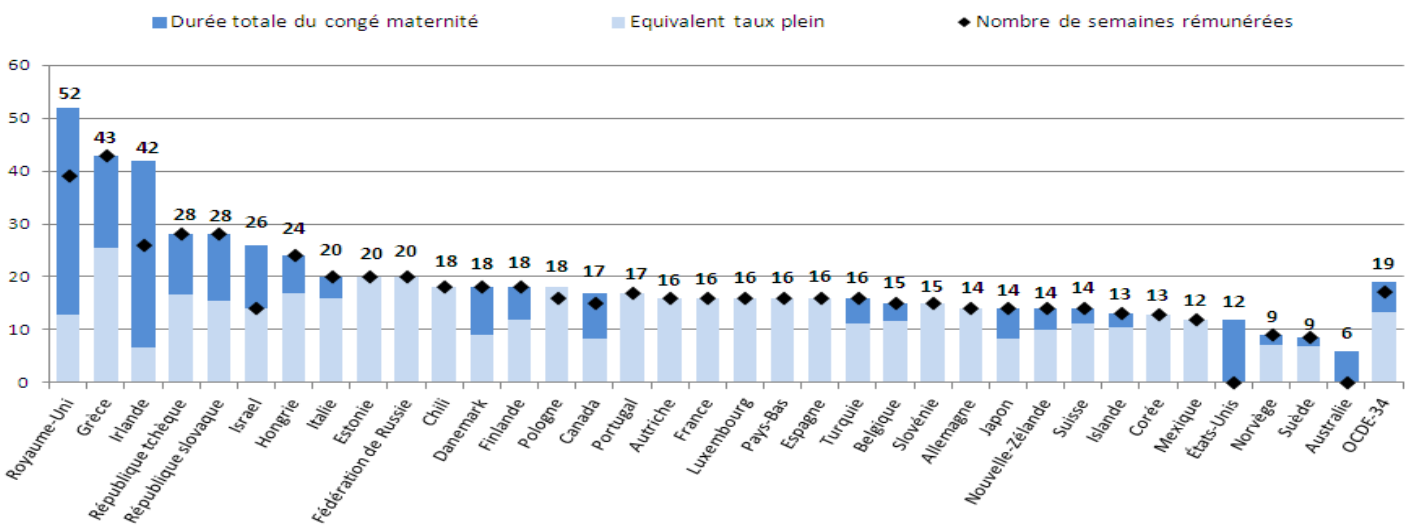

Panel B : Durée en semaines du congé parental ${ }^{2}$, et équivalent taux plein ${ }^{l}$

Eurée totale du congé parental $\quad$ Equivalent taux plein Nombre de semaines rémunérées

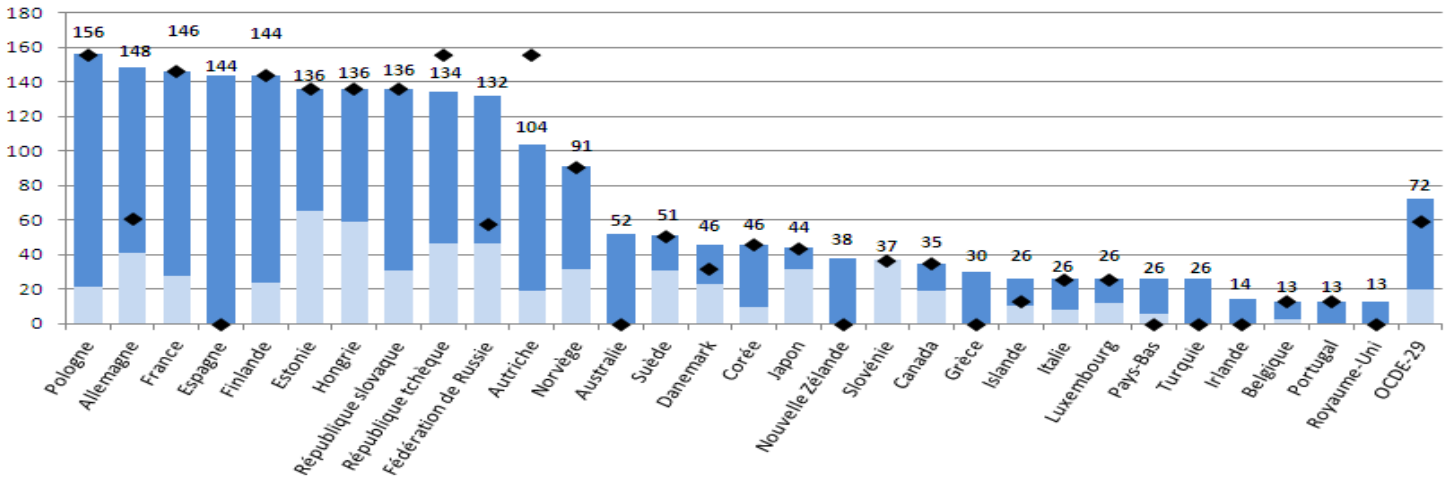

Panel C : Dépenses moyennes en matière de congés par naissance $e^{3}, 2007$ - \% en PIB par habitant

$\%$
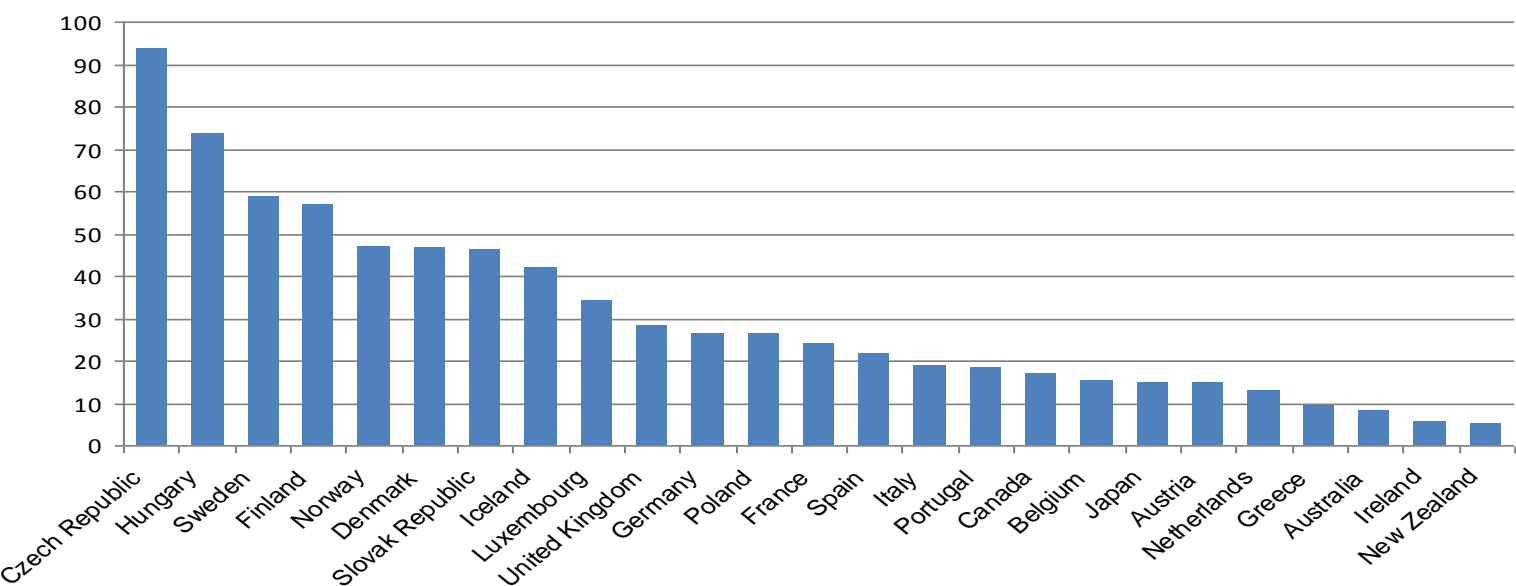

1) L'équivalent taux plein correspond à la durée du congé rapportée à une période d'emploi rémunérée au salaire moyen. Elle mesure ainsi le taux de remplacement global de la période de congé. ETP $=$ durée du congé en semaines*rémunération (en\% du salaire moyen), tel qu'au $1^{\text {er }}$ janvier 2008. Un équivalent taux plein identique peut toutefois correspondre à des combinaisons de durée et taux de rémunérations très différentes entre les pays. La valeur du CLCA a été prise en compte ici pour estimer l'équivalent temps plein en France.

2) La durée considérée correspond à celle pouvant être prise par la mère à l'issue du congé maternité. Les durées du congé maternité et du congé parental peuvent donc s'additionner ici.

3) Les dépenses de congés regroupes celles réalisées au titre des congés maternité, paternité et parental. Les allocations versées à l'occasion de la naissance d'un enfant sont aussi incluses dans cette somme. 
Au total, il existe en France une diversité des aides quelle que soit la solution de prise en charge adoptée. Toutefois, diversité d'inégalités, puisque que le taux d'effort des familles pour bénéficier de chaque mode de garde varie fortement selon le niveau de revenu (Rapport de la Cour des Comptes, 2008). Le recours effectif aux différents modes de «garde » varie fortement avec le niveau de vie du ménage. Ainsi, pour les parents disposant d'un revenu équivalent à 6 SMICs, l'emploi d'une assistante maternelle est la solution de garde la moins onéreuse. A l'inverse, l'accueil au sein un centre collectif est la situation la plus économique pour les ménages dotés d'un revenu équivalent au SMIC. Autrement dit, le «choix » du mode de garde est une décision qui demeure très contrainte par le niveau de revenu du ménage et qui de ce fait demeure très économiquement stratifié : aussi $13 \%$ des enfants de ménages appartenant au dernier quintile de revenus sont, par exemple, gardés par une assistante maternelle, alors que la proportion correspondante n'est que de $3 \%$ pour les premiers et deuxième quintile (Bressé et Galtier, 2006).

Le recours à l'allocation de garde parentale est lui aussi très fortement déterminé par les contraintes financières et la qualité de l'emploi occupé avant la naissance. Parmi les 363000 bénéficiaires du CLCA à taux plein fin de 2008, 40\% auraient préféré continuer à travailler ; mais ils n'ont pas pu le faire parce que leurs conditions de travail étaient trop contraignantes et qu'ils ne trouvaient pas de mode de garde satisfaisant pour leur enfant (HCF, 2010). Les ménages à revenus «modestes » sont évidemment plus directement incités à y recourir puisque le montant du CLCA leur assure un revenu de remplacement plus proche du salaire auquel renoncent et que ce montant n'est pas très différent de celui obtenu par les aides en faveur d'autres modes de garde (Bechtel et al. 2005; HCF, 2010) ${ }^{3}$. Sur l'ensemble des mères de trois enfants en $2007,42 \%$ ne travaillent pas, dont $12 \%$ (265000) sont en congé parental (Crenner, 2011). 76\% d'entre elles ont un niveau de vie au plus égal à celui du deuxième quartile. En outre, les femmes qui ont opté pour un congé parental (ou l'inactivité) occupait plus fréquemment un emploi à horaires atypiques (64\%) que celle qui ont repris un emploi (45\%). Par ailleurs, la plupart des bénéficiaires de l'allocation (70\%) quittent le dispositif parce que la durée maximale de perception est atteinte (Legendre et al. 2011).

Le retour à l'emploi à l'issue d'une interruption complète s'effectue pour $62 \%$ des parents dans les quelques mois après la fin de la prestation, le plus souvent à une durée hebdomadaire similaire à celle effectuée avant la naissance. Pour les trois quarts d'entre eux le retour à l'emploi s'effectue dans le même emploi que celui qu'ils occupaient avant la naissance, un quart ne bénéficiant toutefois pas de la protection donnée par un congé parental. Néanmoins, plus d'un cinquième (22\%) des femmes qui travaillait à temps plein avant la naissance optent pour un temps partiel. En outre, la grande majorité des anciens bénéficiaires de l'allocation (69\%) qui ne travaillent pas quelques mois après son expiration avancent des difficultés à retrouver un emploi, mais ils sont aussi un cinquième à enchaîner sur une autre allocation de même type en raison de la naissance d'un autre enfant ${ }^{4}$.

Au total, la diversification des aides à différents modes de prise en charge de la petite enfance permet en théorie aux parents de sélectionner la solution la plus conforme à leurs préférences. Les points forts de cette diversité sont, d'une part, la complémentarité des aides financières facilitant le recours aux diverses solutions de prise en charge, et l'investissement réalisé dans le développement des services d'accueil. La continuité des aides, sans rupture au cours des années suivant la naissance d'un enfant est, d'autre part, un atout qui permet à beaucoup de parents d'adapter leurs solutions de garde aux besoins qui évoluent dans le temps et avec l'âge des enfants. L'accès à des modes de garde alternatifs

\footnotetext{
${ }^{3}$ Le HCF (2010) note ainsi que le taux de remplacement (revenu du ménage en cas d'inactivité avec CLCA taux plein et aide au logement / revenu en cas d'inactivité, net de frais de garde) varie entre $75 \%$ (cas d'un couple où l'un des membres perçoit 1 SMIC, et l'autre interrompt son activité) 0,8 SMIC) et $50 \%$ (cas d'un couple où l'un des membres perçoit 1,5 SMIC et l'autre interrompt une activité rémunérée à $1 \mathrm{SMIC}$ ).

${ }^{4}$ D'autres évaluations ont mis en évidence l'effet induit par l'extension de l'Allocation Parentale d'Education au deuxième enfant en 1994. Piketty (2005) a, par exemple, estimé qu'environ 110,000 femmes auraient été encouragées à interrompre leur activité lors de cette extension. Sur long terme, il semble que la progression du taux d'emploi des mères de deux enfants ait été ralentie par l'effet de cette allocation, par comparaison à la progression observée pour les mères de un ou trois enfants (Thévenon, 2009).
} 
à la garde parental est ici possible à un âge relativement précoce, ce qui ressort ici comme un caractère assez spécifique à la France, et une composante importante de ses résultats globalement satisfaisants.

Toutefois, le «choix » du mode de garde continue de dépendre très fortement du niveau de revenu du ménage, et des conditions d'emploi qui permettent ou non aux parents de concilier emploi et vie familiale de façon satisfaisante. Le système français est constitué d'une certaine forme de dualité entre une aide à la garde parentale et des aides aux modes extra-familiaux qui bénéficie de façon différenciée aux deux bords de l'échelle sociale. D'un côté, les aides aux solutions de garde extrafamiliales sont plus favorable aux ménages relativement aisés, et où la femme ne fait pas toujours face à des contraintes d'horaires professionnels particuliers; de l'autre, l'aide à la garde parentale peut inciter les mères aux ressources et niveau de salaire plus modestes, connaissant une période de chômage et/ou confrontées à des horaires de travail atypiques à interrompre leur activité pour prendre en charge leur enfant. La perception de cette allocation offre ainsi à ces femmes une parenthèse dans leur vie professionnelle, mais le retour à l'emploi qui peut s'effectuer après plusieurs années d'interruption n'est pas exempt de difficultés. Pour résumer, la dualité du système d'aides à l'accueil des enfants, renforce au lieu de réduire les inégalités d'insertion professionnelles qui préexistent à la naissance d'enfants. Ces inégalités sont, de surcroît, sexuées puisque l'immense majorité (96\%) des bénéficiaires de l'allocation parentale en 2008 sont des femmes, les hommes se bornant le plus souvent aux 11 jours de congé deux tiers des pères tirent ainsi avantage de ces deux semaines qui ne sont sans commune mesure avec les 156 semaines de «congé » parental que prennent souvent les mères de deux ou trois enfants.

En outre, les évolutions de ces dix dernières années sont marquées à la fois par l'augmentation des naissances et la réduction de l'accueil en milieu préscolaire des enfants de moins de trois ans qui imposent une tension supplémentaire sur l'offre d'accueil. Bien qu'en expansion, cette offre permet de compenser la baisse de l'accueil des enfants de moins de trois ans, mais pas «d'absorber » à ce rythme pas «d'absorber» les naissances supplémentaires qui ont lieu chaque année. L'évolution récente est ainsi d'abord marquée par un accroissement nombre de bénéficiaires de prestations d'accueil individuel, de 550,000 en 2000 à environ 720,000 en 2009, tandis que l'accueil en établissement d'accueil n'a lui augmenté que d'environ 50,000 places (de 301,000 à 352,000). Le nombre de bénéficiaires de l'allocation parentale a quant à lui significativement, de 500,000 en 2000 à environ 580,000 en 2009. Autrement dit, tout se passe comme si la réduction de l'accueil des enfants de moins de trois ans en milieu préscolaire (-120,000 places) a été compensée par l'élargissement de l'accueil individuel ou collectif $(+220,000)$, sans toutefois croître au même rythme que les naissances $(60,000$ par an). A défaut d'une augmentation suffisante de l'offre d'accueil, le risque est de voir s'accélérer le nombre de bénéficiaires de l'allocation parental, par défaut plus que par choix véritable, et de voir se renforcer les inégalités d'accès aux différents modes de garde selon le niveau de vie économique et le contexte d'emploi.

\section{Quelles perspectives pour mieux concilier vie familiale et vie professionnelle en France ?}

La grande diversité des modes d'accueil en France et leur grande couverture comparativement aux autres pays est l'une des raisons pour laquelle une large majorité de parents qui travaillent affiche une relative satisfaction vis-à-vis de l'équilibre entre travail et vie familiale en France : près de $81 \%$ des hommes et des femmes, sans écart majeurs entre ces populations, se déclarent satisfait à propos de cet

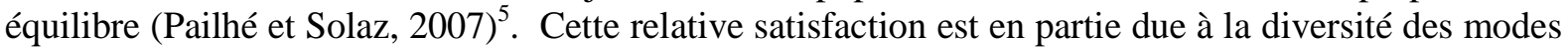
d'accueil offerte en France, qui permet une relative adaptation des modes d'accueil aux contraintes

\footnotetext{
5 Pour autant, les enquêtes de l'Eurobaromètre montre que les Français dans leur ensemble expriment plus souvent que dans d'autres pays européens des difficultés à concilier travail et vie familiale. Cette opinion est paradoxale au vu des conditions objectives plus favorables à la conciliation emploi-famille en France que dans de nombreux autres pays européens. Le renforcement des solutions de garde «flexibles » est alors désigné comme la priorité devant améliorer l'équilibre entre travail et vie familiale (Eurobaromètre, 2008).
} 
professionnelles des parents. Cette diversité répond aussi à la variété des préférences exprimées du point de vue du bien-être de l'enfant et de son développement émotionnel ou cognitif. Aussi, le recours à des nourrices ou assistantes maternelles gardant l'enfant à domicile est la solution jugée la plus satisfaisante parmi les parents qui travaillent, particulièrement en présence d'un enfant en basâge, en raison de la souplesse horaire de ce mode de prise en charge (Kesteman, 2009). Mais, la fréquentation de structures collectives par les enfants dès leur jeune âge est perçue comme un élément favorable à leur « socialisation », leur apprentissage de règles collectives et leur éveil cognitif.

Lorsque les Français sont interrogés sur le type de service à privilégier pour une expansion de l'offre d'accueil, une majorité (61\%) souhaite un accroissement des places en en crèches ou établissement collectif comme premier ou second choix parmi diverses options ${ }^{6}$ (Kesteman, 2009). A ceci, se combinent des opinions plus mitigées concernant l'opportunité de modifier le format du congé parental. Pour près d'un enquêté sur deux (49\%) préfère la situation actuelle (un congé de trois ans, rémunéré de façon forfaitaire à hauteur, environ, de 500 euros par mois). L'autre moitié $(49 \%, 2 \%$ ne se prononçant pas) souhaiterait au contraire la mise en œuvre d'un congé plus court mais mieux indemnisé . $^{7}$.

Dans cette situation, deux solutions sont envisagées dans un récent rapport du Haut Conseil à la Famille (HCF, 2010). La première envisage de compléter les dispositifs existant par la possibilité de recourir à un congé court et rémunéré - comme cela existe déjà et uniquement pour le $3^{\mathrm{e}}$ enfant ; l'autre envisage le remplacement du dispositif de CLCA actuel par la seule option d'un congé court. D'autres instances, comme l'IGAS et l'OCDE (2011) envisagent plus explicitement les avantages liés à l'introduction d'un congé rémunéré au prorata du salaire perçu, et d'une durée plus courte que l'actuel dispositif pour promouvoir un partage plus équilibré de la prise en charge des jeunes enfant entre pères et mères. Cette option pourrait permettre à certaines femmes de ne pas devoir «choisir» entre carrières et enfants, et semble aussi un format de congé nécessaire pour inciter les pères à y avoir recours. Une réduction des inégalités entre hommes et femmes, et entre femmes peut en être attendu de façon ultime, à condition d'être accompagné d'une expansion de l'offre de services d'accueil disponible à l'expiration des congés individuels.

Dans cette section, nous présentons d'abord les raisons qui motivent une réforme dans ce sens. Celleci peut être favorable à l'égalité entre les sexes, probablement sans nuire à la fécondité ni aux enfants. Les expériences étrangères montrent en outre que de telles réformes, sans produire de révolution dans la division du travail au sein des ménages a des effets notables sur les comportements. Enfin, nous envisageons le scénario d'une expansion des modes d'accueil en France nécessaire en cas de raccourcissement du congé parental, et évaluons un ordre de grandeur de son coût en tenant compte des différents modes d'accueil pouvant être favorisés durant la petite enfance.

\subsection{Les justifications d'une réforme}

\subsubsection{Un scénario favorable à la conciliation entre travail et vie familiale et l'égalité des hommes et des femmes en emploi}

La réduction des inégalités d'accès aux différents modes de garde (qui se traduisent aussi en inégalités d'accès à l'emploi pour les femmes) ne peut sans doute être obtenue que par la combinaison de différents leviers d'action. Le premier concerne les aides financières accordées aux ménages pour solvabiliser le recours aux différents modes d'accueil. Dans cette perspective, la Cour des Comptes invite en 2008 à poursuivre la tendance à la réduction de l'effort financier que les familles à bas revenu doivent supporter pour recourir à une assistante maternelle, tendance amorcée par la réforme

\footnotetext{
${ }^{6}$ Ce chiffre est issu du baromètre annuel sur « les conditions de vie et aspirations des Français » réalisé par le Credoc entre décembre 2008 et janvier 2009 auprès d'un échantillon représentatif de 2006 personnes.

${ }^{7}$ Mais les préférences semblent dépendre de l'âge du répondant ou du nombre réel ou souhaité d'enfants. Le principe d'un congé long est préféré par les femmes au foyer $(61 \%$ ), les plus de 60 ans (à un peu moins de 60 $\%)$, les chargés de familles nombreuses $(56 \%)$ et les personnes dont le modèle familial idéal compte au moins trois enfants (plus de $54 \%$ ) ainsi que celles ayant les plus bas revenus (à $54 \%$ ). Les ouvriers $(57 \%$ ), les parents d'un seul enfant $(57 \%)$ et les moins de 40 ans $(54 \%)$ préfèrent un congé raccourci et mieux indemnisé (Kesteman, 2009).
} 
de 2004 et qui peut être amplifiée de manière à réduire les écarts de taux d'effort qui subsistent selon le niveau de vie (Cour des Comptes, 2008). Ce mode d'accueil apportant une souplesse horaire plus grande que beaucoup d'autres modes, l'élargissement de leur accès pourrait donc permettre à certains ménages de parvenir à un équilibre entre travail et vie familiale plus satisfaisant.

Le développement supplémentaire de l'offre de services d'accueil est également encouragé par plusieurs instances, dont récemment le Haut Conseil à la Famille qui met en avant le consensus de ses membres autour de cette question (HCF, 2010x). Le conseil insiste alors sur la nécessité d'une création «nette » de place, qui ne serait pas annulée par la réduction de la préscolarisation des enfants de moins de trois ans.

D'autres instances ont proposé que cet effort supplémentaire en faveur de l'offre de service soit accompagné d'une réforme des droits actuels au congé parental. Il s'agit, en substance, de limiter la période pendant laquelle les mères peuvent interrompre leur emploi, tout en incitant les pères à avoir plus souvent recours pour compenser, au moins partiellement, la réduction de la période de congé prise par les mères.

Les expériences de pays ayant choisi cette voie montre qu'un congé individuel court, non cessible au conjoint, a des effets sur la prise de jours de congés par les pères pour s'occuper des jeunes enfants. Des réformes récentes montrent ainsi que certaines conditions peuvent induire une augmentation du recours au congé par les pères (encadré 2). Ces réformes se montrent efficaces pour initier des changements de comportement, mais elles ne suffisent pas pour véritablement remettre en cause la division du travail qui s'opère à la naissance d'enfants. Ainsi, un déséquilibre subsiste dans la répartition des jours de congé entre pères et mères. Mais le déséquilibre est encore plus fort lorsque l'on considère la répartition du travail non-rémunéré entre hommes et femmes, quel que soit leur situation familiale. Le graphique 5 montre l'ampleur de cette différence, et montre aussi que l'écart en temps de travail non rémunéré entre femmes et hommes tend à diminuer lorsque le taux d'emploi des femmes est plus élevé, les femmes passant en moyenne moins de temps dans ces activités alors que les hommes y investissent plus de temps en moyenne. Bien qu'on ne puisse identifier lequel des ces deux termes est cause ou conséquence, une participation des femmes à l'emploi plus fréquente semble, par conséquent, aller de paire avec un plus grand partage entre hommes et femmes du travail non rémunéré. L'engagement plus massif ou plus continu des femmes dans l'emploi semble ainsi requérir une répartition plus égalitaire du travail non rémunéré.

\section{Graphique 5 : Temps de travail non rémunéré des hommes et des femmes en fonction du taux d'emploi féminin}

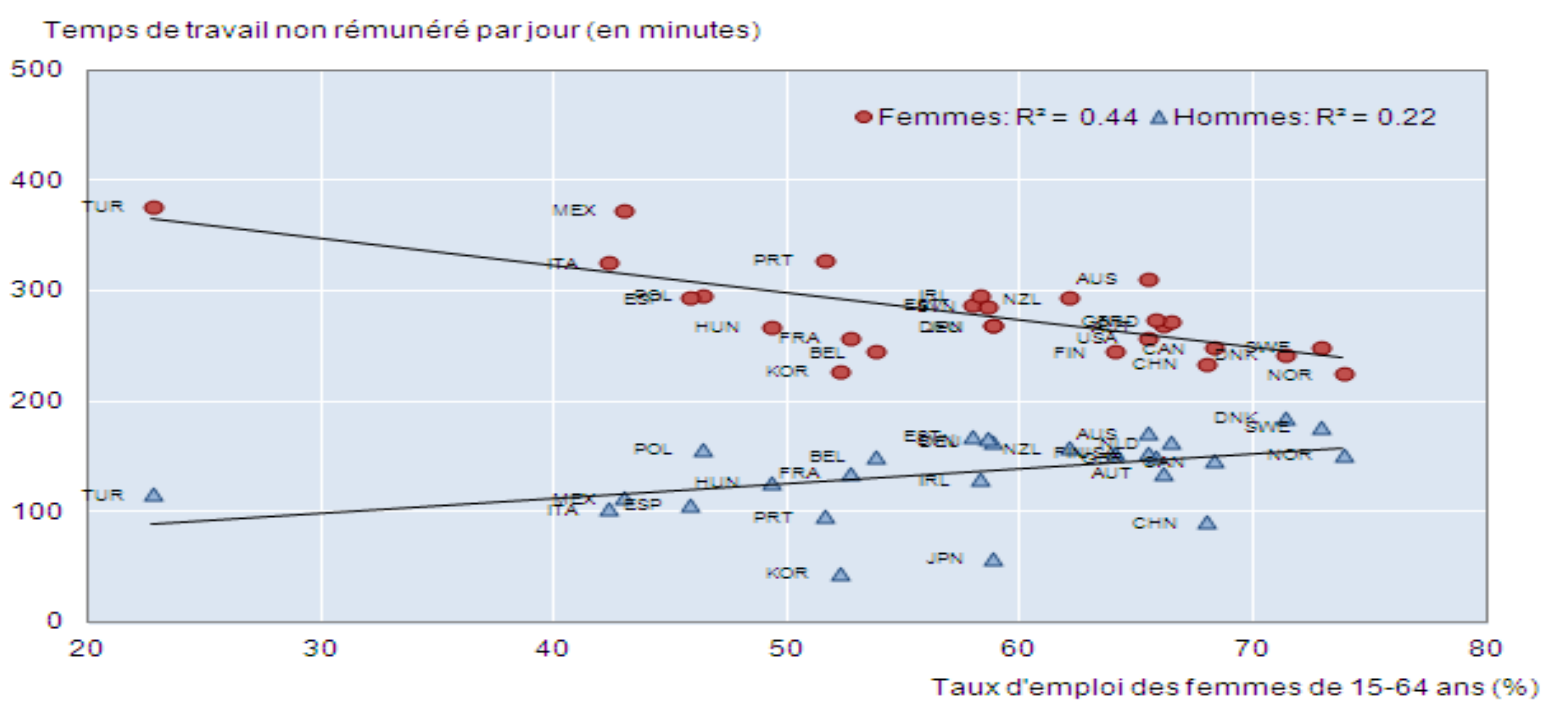

Source: Miranda (2011). CAN : Canada ; LUX : Luxembourg ; DNK : Danemark ; FRA : France ; SWE : Suède ; HUN : Hongrie ; AUS : Australie ; CHN : Chian ; GBR : Royaume-Uni ; BEL : Belgique; IRL : Irlande ; AUT : Autriche ; EST: Estonie ; FIN : Finlande ; DEU : Allemagne ; JPN : Japon ; KOR : Corée du Sud ; NOR : Norvège ; NLD : Pays-Bas ; PRT : Portugal ; POL : Pologne ; CH : Suisse ; ITA : Italie ; USA : Etats-Unis ; MEX : Mexique. 
Plusieurs caractéristiques du paysage institutionnel concourent à la réalisation d'un tel équilibre plus « égalitaire ». L'intérêt financier pour un ménage d'avoir deux actifs, l'accessibilité financière et géographique des modes de prise en charge des enfants, l'accès à des services d'accueil périscolaire, et, pour les parents de très jeunes enfants, le partage des périodes de congés octroyés après la naissance d'un enfant.

La durée totale pour laquelle chaque parent (et en particulier les mères) peut interrompre leur emploi est un facteur déterminant de l'équilibre atteint en termes d'égalité professionnelle et de contribution aux activités parentales. Ainsi, les longues interruptions d'emploi qui suivent une naissance sont assorties de pénalités en termes de niveau de salaire et de progression des carrières. Pour le cas français, Meurs et al. (2010) estime que la période d'interruption d'activité liée à la présence d'enfant (dans le cadre d'un congé parental ou non) et la moindre expérience acquise par conséquent dans l'emploi sont la principale cause des différences de salaire existant entre mères de familles et les femmes sans enfant. Elles n'identifient cependant pas de différences significatives de "rendement" salarial de l'expérience entre ces deux groups de femmes, à la différence de Lequien (2010) qui estime que la réforme de l'Allocation Parentale d'Education de 1994 a induit pour ses bénéficiaires une pénalité dans l'évolution de la rémunération qui persiste dans le temps. Cet auteur estime ainsi par que l'extension de l'Allocation parentale d'Education permettant aux mères de deux enfants d'interrompre leur activité pendant trois ans a produit un ralentissement de la croissance annuelle de salaire pour les salariées qui ont eu un deuxième enfant après la réforme, par comparaison avec celles qui ont eu leur enfant juste avant. Ces différences persistent en outre dix ans après. Aussi, chaque année d'interruption semble avoir produit une baisse de salaire de $10 \%$ après reprise d'emploi.

La littérature internationale confirme très largement l'existence de telles pénalités dans les niveaux et progression de salaire féminin due à une longue interruption d'activité liée la présence d'enfant (OCDE, 2011 pour une revue). Eversston et Duvander (2010) montre que cela est aussi le cas en Suède où le départ en congé parental pour une période de 16 mois affecte négativement les profils de gains salariaux. Le retour à l'emploi chez le même employeur permet d'atténuer les effets dans certains cas où les congés sont relativement courts comme au Canada (35 semaines avec toutefois des variations selon les provinces) où Zhang (2010) estime que l'interruption d'activité induit de fortes pénalités salariales à court terme (i.e. dans les deux premières années), mais que l'écart de salaire entre les femmes qui n'ont pas interrompu leur emploi et celles qui sont revenus chez le même employeur après une interruption s'amenuise dans le temps pour disparaître totalement au bout de sept années. A partir de données internationales, Jaumotte (2003) trouve, quant à elle, que toute augmentation de la durée du congé supérieure à une vingtaine de semaines en équivalent temps plein a un effet marginal négatif sur le niveau de salaire moyen. Autrement dit, l'existence de pénalités persistantes sur l'évolution des salaires et de la carrière semble d'autant plus probable que l'interruption d'emploi dans le cadre d'un congé ou non est longue. Un éloignement de l'activité professionnelle inférieur à une année permet de limiter ce risque. L'enjeu est de définir une durée de congé qui soit suffisante pour permettre aux parents de s'occuper pleinement de leur enfant en bas-âge et de trouver un relais satisfaisant sans quoi ils ne seraient pas en mesure de retourner en emploi à l'expiration du congé ; mais il est aussi de limiter sa durée pour ne pas perturber de façon permanente le fonctionnement de l'entreprise, et ne pas induire des différences de traitements marquées entre salariés selon leur usage ou non des droits à congé.

Pour le cas de la France, un raccourcissement des droits à congé permettant une absence de l'emploi pendant une période de 6 à 18 mois est un scénario qui peut être envisagé pour limiter les effets «pervers» d'une longue interruption, effets qui ne résultent pas d'une décision prise en parfaite connaissance des conséquences. Le raccourcissement des droits pour chaque parent peut être accompagné par un recours plus fréquent des pères à ce dispositif de manière à ce que la réduction de la période de congé prise par la mère puisse être partiellement compensée par une période bénéficiant au père. Certaines caractéristiques des droits à congé peuvent favoriser ce partage, dans certaines limites que les pays les plus avancés sur cette question n'ont toutefois pas totalement levé (Encadré 2). Quel que soit l'ajustement réalisé par les deux parents, un raccourcissement des droits à congé 
devra être accompagné par un accroissement de l'offre d'accueil de façon à ce que la prise en charge de l'enfant soit la plus continue possible, sans période pendant laquelle les parents n'auraient d'autres alternatives à leur propre prise en charge ou aux moyens informels.

\section{Encadré 2 : Exemples de la diversité des systèmes de congés en cas de naissance}

Les droits au congé de maternité (ou de grossesse), de paternité et parental assurent une protection de l'emploi aux parents qui quittent leur travail pour s'occuper de leurs enfants en bas âge. Cette période est souvent couverte par des compensations de revenu publiques, qui peuvent être complétées par les employeurs. Dans certains pays, le congé parental est complété par une période de congé supplémentaire (congé de garde à domicile/congé d'éducation) que les parents peuvent prendre pour s'occuper d'un jeune enfant souvent jusqu'à l'âge de 3 ans. Pendant cette période, la garantie de revenu est bien moindre que pendant les périodes de congé de maternité/congé parental.

Face à cette diversité, on peut distinguer au moins trois logiques :

- Une logique de congé minimal dans les pays anglo-saxons où ce congé est de durée très courte, non ou faiblement rémunéré ;

- Une logique de congé de durée relativement limitée (de 12 à 16 mois, rémunéré au prorata du salaire au Danemark, en Islande, Suède, en Estonie ou depuis récemment l'Allemagne dans les pays nordiques;

- Une logique de congé pour une période beaucoup plus longue, combinée à une rémunération forfaitaire nettement inférieure au salaire moyen, comme en France. Certains pays comme la Norvège ou la Finlande combinent un congé parental relativement court (54 semaines dans le premier cas et 26 dans le second) rémunéré au prorata du salaire, avec le versement d'une allocation forfaitaire aux parents désireux de prolonger leur interruption d'activité jusqu'au troisième anniversaire de l'enfant.

L'incitation au recours au congé par les pères est aussi un paramètre qui distingue les pays offrant un congé relativement court mais bien rémunéré. Ce schéma s'appuie donc sur une vision intégrée entre la promotion de l'emploi, de l'égalité entre hommes et femmes et du partage de l'éducation des enfants par les parents. Au total, l'expérience internationale suggère que le recours au congé par les pères est plus probable dans les conditions suivantes (OCDE, 2011):

- L'existence de droits strictement individuels en matière de congé et de rémunération lui étant, non transférable au conjoint. rémunération juridiquement liée au congé devrait par conséquent être substituée au CLCA qui est aujourd'hui un droit familial. Différentes formes quota de congé parental réservé à l'usage exclusif chaque parent. La proposition de l'IGAS d'introduire un "congé d'accueil de l'enfant" de 8 semaines, à partager à égalité entre les deux parents s'inscrit dans cette perspective.

- Une rémunération proportionnelle au salaire (du moins jusqu'à un certain plafond) semble aussi une condition nécessaire pour inciter non seulement les pères à y avoir recours, mais aussi les mères percevant un salaire relativement élevé. La rémunération à hauteur de $60 \%$ proposée par l'IGAS rentre aussi dans ce care, mais est inférieur au taux appliqué en Allemagne ou Suède $(67 \%)$ ou en Islande $(80 \%)^{8}$.

- D'autres incitations peuvent aussi être associées pour encourager le partage de la période de congé entre les deux parents. Par exemple, plusieurs pays octroient une période de congé supplémentaire lorsque les deux parents ont recours à un minimum de semaines de congé. Deux mois de congé supplémentaires sont, par exemple, octroyés si chaque parent prend un congé d'une période minimale de deux mois en Allemagne. Le Portugal propose depuis 2009 une formule innovante en proposant une rémunération à taux plein de $100 \%$ du salaire pour l'ensemble de la période de congé si les parents prennent un minimum de 30 jours de congés sur une période totale de 150 jours. Le taux de rémunération est de $80 \%$ au cas où le quota d'égalité n'est pas respecté 9 .

- La possibilité de prendre le congé à temps partiel ou par périodes séparées semblent aussi permettre une adaptation aux parents qui souhaiteraient ne pas s'éloigner de leur emploi pour une trop longue période.

L'Islande est le pays qui va le plus loin dans l'incitation à un partage égalitaire du congé parental en allouant à chaque parent d'un enfant de moins de 18 mois un droit depuis 2001 à un congé individuel de 13 semaines, rémunéré à $80 \%$ du salaire perçu dans l'emploi. Un congé parental supplémentaire de 13 semaines est proposé avec les mêmes conditions de

\footnotetext{
${ }^{8}$ En toute rigueur, le taux de remplacement effectif dépend du plafond qui sera appliqué à la rémunération du congé (voir Base OCDE sur la Famille, indicateur PF2.4).

${ }^{9}$ Le gouvernement suédois a également introduit en 2008 une incitation fiscale sous la forme d'un «gender equality bonus » qui octroie une réduction de $€ 10$ par jour de congé pris au-delà du quota par le conjoint dont la période de congé est la plus courte. La réduction d'impôt maximale est donc de $€ 1410$ en cas de partage strictement égalitaire. Ce mécanisme ne semble toutefois n'avoir eu que peu d'effet jusqu'à présent Duvander et Johansson (2010).
} 
rémunération, les parents étant libres de choisir sa répartition ${ }^{10}$. Cette réforme induit des changements significatifs dans le temps consacré par les pères à la prise en charge des très jeunes enfants puisque la proportion de jours de congé pris par les pères est passée de $3 \%$ avant la réforme à $35 \%$ aujourd'hui, c'est-à-dire une proportion qui correspond mais n'excède pas leur droit individuel (Eydal et Gislasson, 2008).

Les inégalités de genre demeurent toutefois importantes puisque $90 \%$ des mères utilisent leur droit individuel, tandis le taux de recours par les pères n'est que de $19.7 \%$. De plus, la durée moyenne du congé est de 99 jours pour ces derniers (i.e. la durée de leur droit individuel), tandis la période moyenne de congé des mères est de 185 jours.

La Norvège et la Suède sont aussi deux pays réservant des quotas de congé à l'usage exclusif de chaque parent. En Norvège, ce quota est de 9 semaines pour les mères et 10 semaines pour les pères sur une période totale de congé de 46 semaines. En Suède, le quota est de 60 jours pour chaque parent sur un total de 480 jours. L'introduction de ce quota a induit une augmentation de la participation des pères à la prise en charge du jeune enfant. Les inégalités entre sexe demeurent néanmoins importantes. En Suède, on estime que les pères prennent à peu près 22\% des jours de congé en 2009 (Moss, 2010). En Norvège, $15 \%$ des pères prennent un congé, mais les mères sont présentes dans la très grande majorité des cas où les pères sont en congé. Une réflexion est de ce fait en cours sur l'opportunité de contraindre ou non les parents à prendre des périodes séparées de congé.

L'Allemagne offre une autre illustration puisque ce pays a également réformé son dispositif de congé parental, en passant d'un régime à congé long rémunéré de façon forfaitaire (et sans condition d'activité préalable) à un congé "à la suédoise ». Cinquante deux semaines de congé donnent ainsi droit à une rémunération égale aux deux tiers du salaire net ${ }^{11}$. Il est néanmoins possible de l'utiliser sur 24 mois avec une rémunération à taux réduit. Par ailleurs, un bonus de deux mois de congé est accordé si le père prend au moins deux mois de congé sur le total de 14 mois. Dans ce contexte, le taux de recours des pères s'est accru depuis la mise en place du dispositif, de 3.5\% des pères éligibles au dernier trimestre 2006 à $14.3 \%$ au premier trimestre 2008. Alors que moins de $9 \%$ des enfants nés avant cette réforme avaient un père prenant un congé parental, cette proportion a été plus doublé (17\%) en 2008 (Office Statistique Fédéral, 2010).

Plus récemment encore, en 2009, le Portugal a réformé son système de congé avec 150 jours de congés offerts aux parents, et 30 jours de bonus supplémentaire si chacun d'eux prend effectivement au moins 30 jours. La proportion de pères prenant un congé est alors passée de moins de $1 \%$ à $17 \%$ en 2010. En plus de ce congé initial, chaque parent peut bénéficier de trois mois de congé supplémentaire beaucoup plus faiblement rémunéré.

Contrairement aux pays précédents, le Danemark est caractérisé par congé relativement court (32 semaines de congé parental qui s'ajoutent aux 18 semaines de congé maternité), rémunéré à un taux de remplacement de $100 \%$ pour les salaires inférieurs au salaire moyen, mais qui décroît rapidement en raison d'un plafond relativement bas. Le congé parental est ici un droit familial sans quota strictement réservé à chaque parent. Les pères bénéficient toutefois de 2 semaines de congé paternité pleinement rémunérées. Dans ce contexte, $94 \%$ des mères font usage du congé parental, tandis que la proportion est de $26 \%$ pour les pères. Mais $89 \%$ de ces derniers ont recours au congé paternité (SFI, 2007). A l'issue des 50 semaines de congé, près de $85 \%$ des enfants sont accueillis en centre d'accueil dès leur deuxième année, alors qu'ils ne sont que $15 \%$ dans leur première année, pour l'année 2005.

\subsubsection{Un effet probablement limité sur la fécondité}

L'opportunité d'une réforme de l'accueil de la petite enfance est à apprécier à l'aune des effets qui peuvent être attendus sur une diversité d'aspects déjà évoqués en première partie. La fécondité est l'un des aspects qu'une réforme des modes d'accueil peut affecter. Un effet limité, voire positif, semble pouvoir être attendu, pour plusieurs raisons. D'abord, les taux de fécondité les plus élevés sont observés aujourd'hui, dans les pays où les taux d'emploi des femmes sont eux-mêmes élevés, contrairement à ce qui était observable il y a quelques décennies (Graphique 6). La combinaison de taux de fécondité et d'emploi des femmes élevés, comme c'est le cas en en France n'est donc pas exceptionnelle, et est observée dans des pays où le contexte institutionnel est très différent. Aussi, «l'excédent » démographique de la France par rapport à ses voisins tient-il, pour une bonne part, à la faible incidence de l'infécondité, dont la fréquence est demeurée globalement stable (ou en très lente augmentation) depuis les générations depuis l'après deuxième guerre mondiale (Breton et Prioux,

\footnotetext{
${ }^{10}$ A l'expiration de cette date, 13 semaines de congé supplémentaires mais non rémunérées dans ce cas peuvent en outre être prises tant que l'enfant n'a pas atteint sa huitième année.

${ }^{11}$ L'usage du congé est aussi relativement flexible : d'abord les parents peuvent prendre un congé partiel et continuer à percevoir une allocation compensatrice si leur activité ne dépasse pas 30 heures par semaines. Par ailleurs, la dernière année du congé peut être prise tant que l'enfant n'a pas atteint son huitième anniversaire. Enfin, les parents peuvent bénéficier du congé simultanément, et prendre le congé en deux périodes au maximum
} 
2009 ; Toulemon et al. 2008). La probabilité d'avoir une famille de plus de deux enfants a quant à elle décru au fil des générations, mais demeure plus forte en France et dans les pays à forte fécondité par comparaison aux pays à fécondité basse. En outre, d'autres pays, comme la Suède, la Norvège ou les pays anglophones, combinent aussi une forte proportion de famille nombreuses, avec trois enfants ou plus, avec des dispositions en matière de congé d'emploi suivant une naissante très différentes.

\section{Graphique 6 : Indice de fécondité et taux d'emploi des femmes}
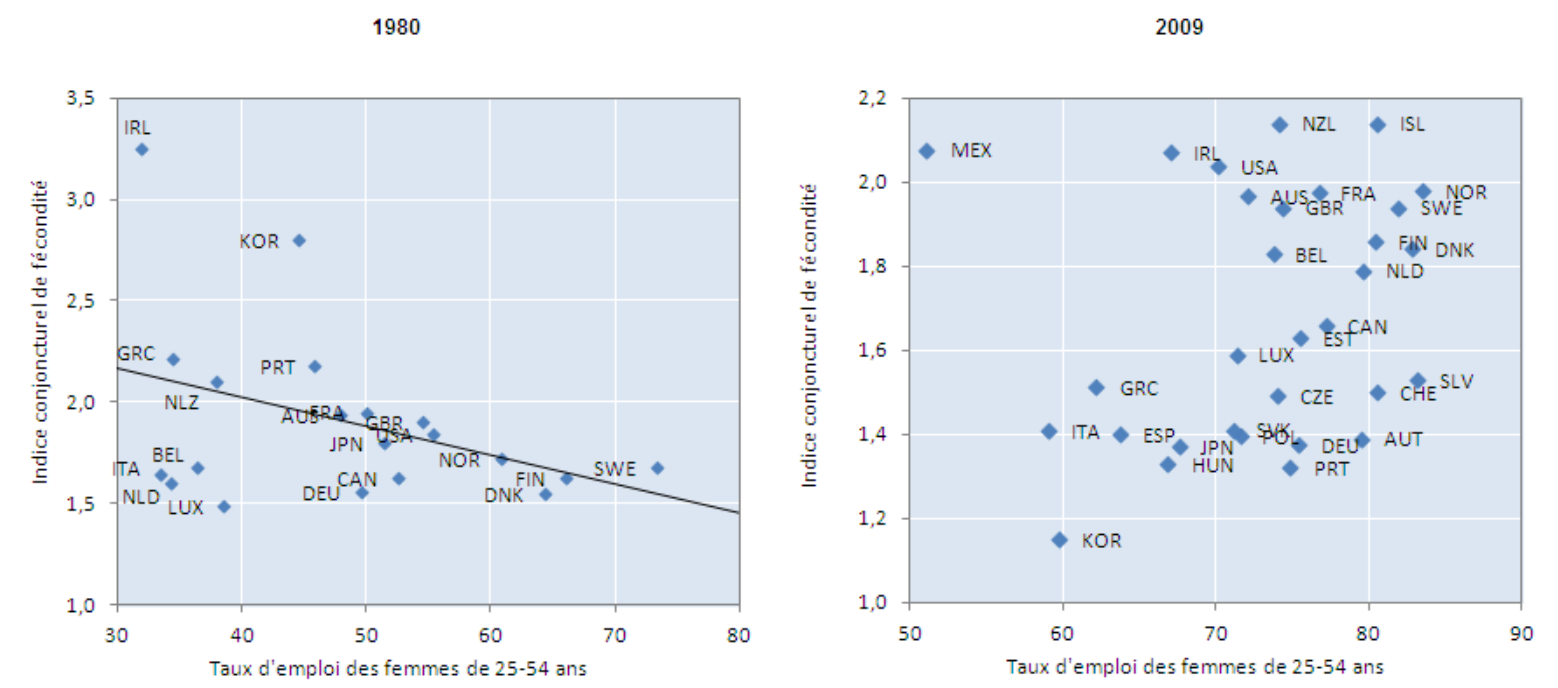

L'axe des y (indice conjoncturel de fécondité) va de 1.0 à 3.5 pour 1980 et de 1.0 à 2.2 pour 2009 . Source : OCDE (2010b), Base de données de POCDE sur la famille, SF2.1.

Par ailleurs, les analyses de l'impact des politiques sur la fécondité suggèrent que les caractéristiques des congés en matière de durée et de rémunération qui leur sont associées directement ou non ont un effet relativement faible sur les taux de fécondité ${ }^{12}$. En revanche, les transferts monétaires qui interviennent plus tard et plus régulièrement dans l'enfance, et surtout par la couverture des services d'accueil des jeunes enfants semble avoir une influence plus forte sur les taux de fécondité (Luci et Thévenon, 2011 ; Thévenon et Gauthier, 2010) ${ }^{13}$. Au total, on peut attendre un effet plus marqué de l'extension des modes d'accueil que celui de l'éventuelle réduction éventuelle de la période de congé qui serait associée à l'augmentation de l'offre d'accueil. En outre, la réforme du congé parental en France ne concernerait que les naissances de rang deux ou supérieur, dont l'occurrence ne risque que d'être faiblement impactée par des changements de durée ou de rémunération du congé ${ }^{14}$.

\subsubsection{Une expansion qui peut aussi bénéficier aux enfants}

\footnotetext{
${ }^{12}$ L'effet semble en outre exercé davantage sur le calendrier d'une naissance programmée que sur la décision d'avoir ou non un enfant (Thévenon et Gauthier, 2010).

${ }^{13}$ En fait, l'effet des congés et des réformes les concernant semble avant tout s'exercer sur le calendrier des naissances, en permettant de ne pas reporter une naissance prévue, plus que sur le nombre total d'enfants que les parents auront au cours de leur vie. Ainsi, les analyses faite sur les pays Nordiques suggèrent que les réformes du conge parental qui ont eu lieu par exemple en Suède ont réduit de façon persistante l'écart de temps existant entre la naissance des deux premiers enfants, mais aucune incidence sur le nombre d'enfant final n'est avéré. Les effets sont, quoi qu'il en soit d'ampleur modeste. Pour le cas de la France, Piketty (2005) estime que l'extension de l'APE aux parents de deux enfants à partir de $1994 \mathrm{a}$ pu expliquer au maximum 20 à $30 \%$ de la croissance des naissances de second rang intervenue entre 1994 et 2001 et au mieux $20 \%$ de l'augmentation des naissances de troisième rang. Cette part explicative est donc limitée, et il n'est pas dit que ces naissances n'auraient pas eu lieu plus tard, à un âge plus avancé.

${ }^{14}$ Certaines analyses conduites dans les pays nordiques suggèrent en outre que la participation du père aux activités parentales suivant la naissance d'un premier enfant influence positivement le calendrier de conception d'un deuxième enfant (Duvander, Lappegard, et Andersson, 2010).
} 
Les conflits éventuels pouvant exister entre l'emploi des parents, l'organisation du temps de travail et des activités parentales et le bien-être des enfants sont aussi l'objet d'une abondante littérature. L'effet d'une reprise d'emploi des mères dans les mois suivant la naissance d'un enfant sur le développement social et cognitif de ce dernier a reçu une attention toute particulière ${ }^{15}$. Plusieurs paramètres interviennent pour apprécier cet effet. D'un côté, l'emploi des parents induit un revenu supplémentaire pour le ménage dont tous les membres du ménage, y compris les enfants, sont susceptibles de bénéficier (Gregg et al. 1999 ; Whiteford and Adema, 2007 ; Esping-Andersen, 2008). D'un autre côté, les parents qui travaillent auront recours à un service extrafamilial pour prendre en charge leur enfant, avec une intensité variable qui dépend de leurs contraintes professionnelles. Le bénéfice qui peut être associé pour l'enfant à cet accueil extra-familial dépend alors de l'âge auquel il intervient, mais aussi et surtout de la qualité du service et de la durée pour laquelle l'accueil extrafamilial est réalisé. L'accueil extra-parental exerce aussi un effet sur le développement de l'enfant qui varie certainement selon type de prise en charge, en milieu familial ou collectif, dans un cadre récréatif ou éducatif.

La décision de reprendre une activité après la naissance d'un enfant s'effectue donc dans un contexte de coûts et bénéfices qui sont difficiles à tous mesurer mais qui sont d'autant plus importants que les revenus d'un ménage sont faibles. Certaines analyses ont pu pointer l'existence d'association négative entre l'emploi des mères et certains aspects du bien-être de l'enfant, mais une revue de littérature suggère que de telles associations sont circonscrites à des circonstances ou des configurations de ménages particulières. Une relation positive entre l'emploi des mères et le bien-être des enfants est plus généralement identifiée. Une analyse récente de Huerta et al. (2011) basée sur une exploitation de cohortes d'enfants de cinq pays (Australie, Canada, Danemark, Royaume-Uni et Etats-Unis ${ }^{16}$ ) souligne les résultats suivants, qui sont aussi confirmés par d'autres revues de littérature ${ }^{17}$ :

- Les performances cognitives ${ }^{18}$ des enfants peuvent être affectés négativement lorsque le retour à l'emploi intervient quelques mois après la naissance (dans les 6 mois), particulièrement lorsqu'elles travaillent à temps plein ; mais l'association identifiée est de faible magnitude et n'est pas observée dans tous les contextes: cette relation négative apparaît au Royaume-Uni ou dans certains cas aux Etats-Unis, tandis que les associations sont plus fréquemment positives au Danemark, Canada ou en Australie.

- La faible association négative observée entre l'emploi des mères avant six mois et différentes dimensions du développement de l'enfant ne sont observés dans les familles à faible niveau de revenu et faible capital éducatif. Il semble ainsi que ces parents aient en moyenne moins d'activité stimulant l'éveil de l'enfant, et que par conséquent, la différence de statut au regard de l'emploi ne soit pas un critère très discriminant. En outre, pour ces familles, l'effet strict de l'interaction parent-enfant est plus vraisemblablement compensé par l'influence positive du

\footnotetext{
${ }^{15}$ Cette attention très particulière à l'effet du statut des mères a des raisons idéologiques, principalement, et statistiques, puisque la faible fréquence de pères s'occupant des enfants en bas-âge ne permet pas toujours d'analyser son effet propre sur les enfants par différence avec les enfants dont le père est en emploi.

${ }^{16}$ La plupart des recherches menées aux Etats-Unis soulignent que l'emploi précoce de la mère peut avoir des conséquences négatives sur le développement ou la santé de l'enfant (Han et al., 2001; Brooks-Gunn et al., 2002; Baum, 2003; Belsky, 2004; Ruhm, 2003; Ruhm, 2004; Baker et al., 2005). Toutefois, d'autres études concluent à un effet plus mitigé, ou même à un effet négatif sur le développement de l'enfant lorsque sa prise en charge est exclusivement effectuée par la mère (Bernal and Keane, 2006; Gregg et al., 2005; Stafford, 1987; Waldfogel et al., 2002).

${ }^{17}$ Voir notamment Kamerman (2003) ou OCDE (2009; 2011).

${ }^{18}$ Blasko (2008) recense quant à elle la littérature analysant les relations entre l'emploi des mères et le développement socio émotionnel des enfants. Elle conclut que certaines études peuvent pointer une association négative quand la mère est en emploi lorsque l'enfant est âgé de moins de un an. L'effet est néanmoins souvent observé à court terme, et peut disparaître à long terme. Ruhm (2000) et Tanaka (2005) suggère quant à eux qu'une durée de congé parentale inférieure à 6 mois peut être néfaste pour la santé de l'enfant et sa probabilité de décès. Toutefois, Baldi et Chapple (2011) ne parviennent pas à répliquer leurs résultats à partir d'une base de données plus complète et actualisée.
} 
revenu perçu par l'intermédiaire de l'emploi maternel, voire par le recours à un mode de garde formel.

- Enfin, l'accueil au sein de structures formelles ou en milieu préscolaire parait généralement induire des conséquences positives pour le développement cognitif des enfants, bien que des effets négatifs sur les comportements ou attitudes des enfants puissent être observées lorsque les enfants sont pris en charges par des services de qualité faible ou lorsque que cette prise en charge s'effectue pour des durées quotidiennes très longues (Ruhm, 2004a ; Belsky et al., 2007 ; Stamm, 2009 ; Datta Guptta et Simonsen, 2010 ; Esping-Andersen et al., 2011) ${ }^{19}$.

La France ne dispose pas encore d'une cohorte d'enfants permettant de faire de telles analyses. Toutefois, certaines analyses ont montré sur des échantillons réduits que la scolarisation précoce peut exercer un effet positif sur le développement social et cognitif des enfants. Ainsi Zazzo (1984) remarque dès les années 1980s que les enfants fréquentant les crèches s'adaptent plus facilement à l'école, et sont souvent plus sociables avec leurs camarades même s'ils peuvent aussi se montrer plus agressifs que les autres. Pour Cohen (1995), le cumul des fréquentations de la crèche et de l'école maternelle favorise nettement un parcours scolaire sans incident, en particulier pour les enfants de milieux populaires. L'accueil en crèche facilite aussi l'acquisition de vocabulaire, de bases pour la lecture, la capacité de concentration et la compréhension. L'accès gratuit et relativement précoce à l'éducation préscolaire est ainsi une spécificité forte du système français qui permet aux parents d'accéder à un mode d'accueil peu onéreux, qui peut être bénéfique à la fois pour les enfants et parce qu'il facilite la conciliation d'un emploi avec la vie familiale. Les familles de milieux défavorisés bénéficient ainsi tout particulièrement d'un mode d'accueil financièrement abordable, conciliable avec un emploi et bénéfique au développement et à l'insertion scolaire des enfants.

L'entrée en école maternelle de l'enfant à deux ans plutôt qu'à trois, est aussi un facteur qui peut accélérer cette conciliation. Caillé (2003) estime, par exemple, que les bénéfices d'une préscolarisation intervenant à deux ans sont visibles pour les enfants de catégories sociales défavorisées, même si globalement cet avantage lié à l'entrée à l'école maternelle à 2 ans plutôt qu'à 3 est relativement faible. Plus récemment, Goux et Maurin (2010) renversent la perspective et estiment que l'accès à l'école maternelle à deux ans plutôt qu'à trois n'exerce pas d'effet négatif sur la réussite scolaire postérieure de l'enfant, tandis qu'un effet positif sur la probabilité d'emploi des mères isolées est identifié (aucun effet significatif n'étant en revanche identifié pour les femmes vivant en couple). L'effet de l'accessibilité aux structures préscolaires est alors d'autant plus important pour les mères seules que l'enfant grandit, ceci entraînant une perte d'éligibilité aux autres prestations.

Autrement dit, l'accès à des modes d'accueil extra-familiaux exerce un effet positif sur l'accès des femmes à l'emploi, qui lui-même influence positivement le bien-être matériel des familles et des enfants, avec des répercussions potentiellement positives sur le développement social et cognitif de ces derniers. L'impact sur les enfants dépend néanmoins du type de mode d'accueil qui est au moins couplé à la prise en charge parentale, de la durée de cet accueil, et donc des conditions de travail qui permettent alors aux parents de concilier temps parental et temps de travail dans des conditions favorable au bien-être et l'éducation des enfants. L'accès à l'école maternelle joue un rôle particulier puisqu'il permet une très nette augmentation des taux d'emploi des femmes à temps plein, plus forte

\footnotetext{
${ }^{19}$ La plupart des références sur les expérimentations en matière d'accès à l'éducation préscolaire exhibent des effets positifs sur le développement des enfants et des adolescents (Currie, 2001 ; OCDE, 2011 ; Huerta et al., 2011). Cependant, certaines études pointent une influence négative sur les comportements ou les tests cognitifs d'une fréquentation précoce de centres d'accueil collectif ou jardins d'enfant, (Lefebvre et al., 2011 ; DeCicca et Smith, 2011). La fréquentation massive de structures d'accueil de faible qualité, et la durée quotidienne trop grande pour laquelle s'effectue cette prise en charge sont des hypothèses pouvant expliquer cette observation, mais qui n'ont pu être testées. Néanmoins cette hypothèse est accréditée par Datta Gupta et Simonsen (2010) qui trouvent qu'au Danemark, l'augmentation des heures passées en accueil familial de 30-40 heures çà 40-50 heures hebdomadaires et en établissement préscolaire de 20-30heures à 30-40 heures hebdomadaire a un effet dépressif sur les tests non-cognitifs des enfants.
} 
en France que dans de nombreux pays européens (Thévenon, 2009), cet accès étant particulièrement favorable aux mères en situation de monoparentalité.

\subsection{Réformer l'accueil de la petite enfance en France : quel coût ?}

Dans cette perspective, un accroissement de l'offre de service d'accueil combiné à la possibilité de prendre un congé court mais bien rémunéré peut être un moyen d'élargir l'éventail d'options dont disposent les familles pour concilier travail et vie familiale ${ }^{20}$.

Dans la section qui suit, on se propose d'estimer le coût budgétaire que représenterait à court terme ce type de réforme. On envisage, pour cela, l'accroissement des capacités d'accueil étant nécessaire pour couvrir l'ensemble des jeunes enfants à l'issue d'un congé «parental» qui pourrait varier entre six mois et un an. La question du partage de ce congé entre les parents n'est pas abordée ici, malgré son incidence probable sur le coût. Plusieurs scenarios sont envisagés pour tenir compte de la diversité des modes d'accueil qui pourraient être choisis et " parcours de garde » qui pourraient être privilégiés au cours des six premières années de l'enfant.

Pour estimer le coût (et les bénéfices) d'une réforme de l'accueil de la petite enfance, la situation idéale serait de pouvoir mesurer ses effets sur les décisions en matière de mode de garde, d'une part, les comportements de fécondité et d'offre de travail pour les hommes et les femmes, d'autre part. A défaut de telles modélisations, on raisonne ici par comparaison de scénarios fondés sur différentes hypothèses simplificatrices concernant la demande d'accueil. Ces scénarios donnent un ordre de grandeur du coût maximum impliqué à court terme par une réforme des modes d'accueil. Bien que simplificatrices, ces hypothèses sont raisonnablement réalistes puisqu'elles correspondent à l'expérience d'autres pays. Notons toutefois que cette démarche néglige les bénéfices qui pourraient être rapidement obtenus du fait des rentrées fiscales en cas d'augmentation des taux d'emploi suscité par un accroissement de l'offre de garde. Le coût brut de l'investissement en modes de garde est donc ici estimé, sans considérer le coût ou le bénéfice net qui pourrait en être issu.

Deux jeux d'hypothèses importent pour construire les scénarios requis. Le premier concerne l'enchaînement des différents modes de garde au cours de la petite enfance. Cet enchaînement varie avec la durée du congé parental, le choix du mode de garde adopté à son issue et de l'âge auquel s'effectue l'entrée en école maternelle. Plusieurs scénarios sont envisagés selon le calendrier de transition d'un mode à l'autre. Le scénario « central » correspond au cas où le congé parental est pris pendant la première année, suivie de deux années de prise en charge par un service d'accueil formel, et d'une entrée en école maternelle qui s'effectue à l'âge de trois ans. Le graphique 7 résume alors les 6 scénarios envisagés pour prendre en compte la diversité des parcours possibles ${ }^{21}$.

\footnotetext{
${ }^{20}$ Cette possibilité est discutée dans plusieurs rapports plus ou moins récents, parmi lesquels Brin (2005), Tabarot (2008), Clergeau (2009) et le récent avis du Haut Conseil à la Famille (2010).

${ }^{21}$ Autrement dit, la palette des scénarios possibles nous permet d'envisager des situations où varie la durée du congé et le mode de garde. Ils doivent être considéré comme des cas extrêmes à l'intérieur desquels les comportements peuvent varier. Ces scenarios n'impliquent donc pas l'adoption d'un standard de comportement unique, mais pour but d'estimer des ordres de grandeurs en fonction des comportements qui peuvent varier.
} 
Graphique 7 : Six parcours de garde au cours de la petite enfance

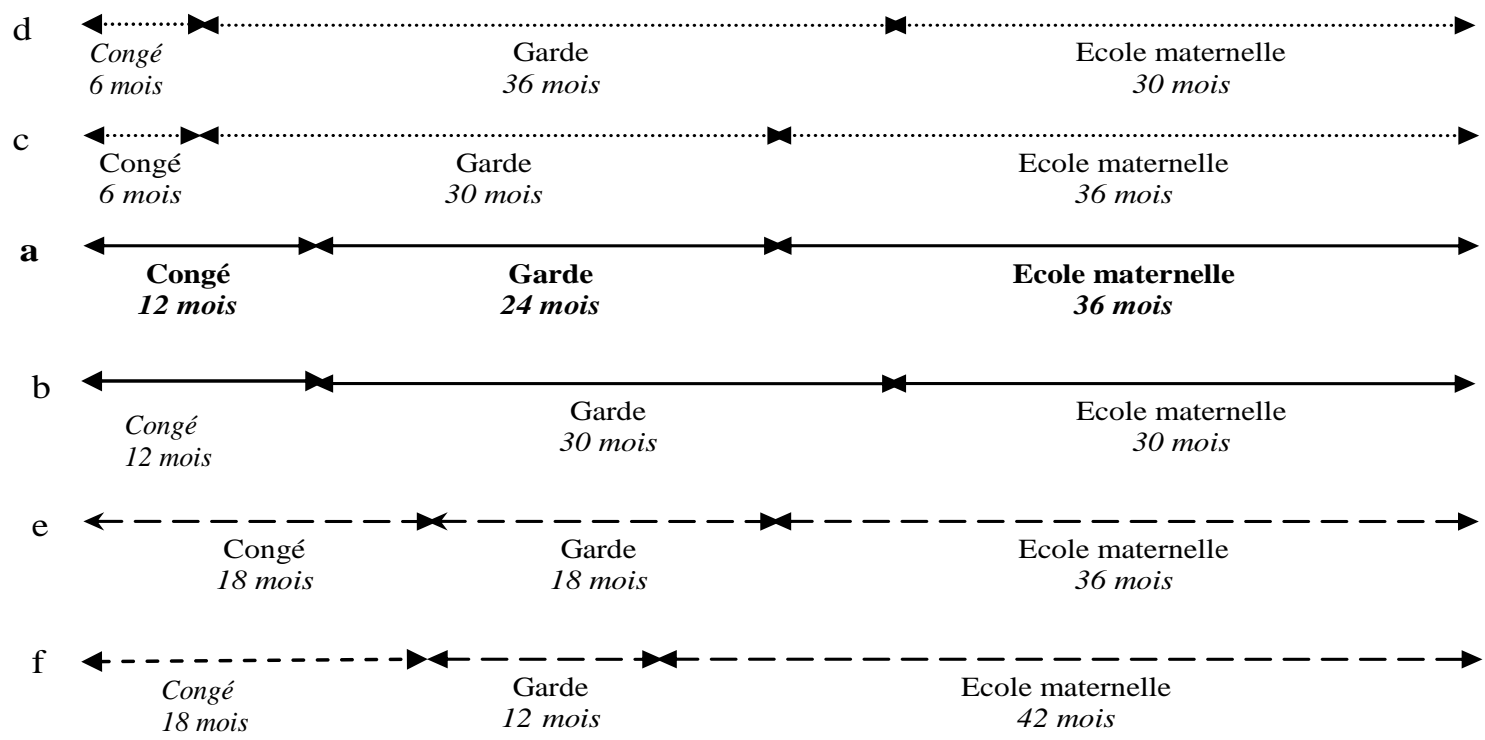

Le deuxième jeu d'hypothèses nécessaires concerne le mode d'accueil qui est privilégié à l'issue du congé et la combinaison qui peut être faite de différents modes d'accueil. La prise en charge par l'État du financement de l'accueil variant fortement selon le mode, le coût public total d'une expansion de l'offre de services varie fortement selon le mode d'accueil privilégié pour cette expansion. Le tableau 2 rappelle l'évaluation faite par la Mission de révision générale des politiques publiques du coût par enfant de chaque mode de garde.

Tableau 2 : Coût annuel moyen des différents modes de garde

\begin{tabular}{|c|c|c|c|c|c|c|}
\cline { 2 - 6 } \multicolumn{1}{c|}{} & $\begin{array}{c}\text { Coût par } \\
\text { enfant }\end{array}$ & $\begin{array}{c}\text { part payée } \\
\text { par les } \\
\text { familles }\end{array}$ & $\begin{array}{c}\text { part } \\
\text { payee } \\
\text { par l'Etat }\end{array}$ & $\begin{array}{c}\text { part payee } \\
\text { par les } \\
\text { CAF }\end{array}$ & $\begin{array}{c}\text { Part } \\
\text { gestionnair } \\
\text { e } \\
\text { collectivité } \\
\text { locale ou } \\
\text { autre }\end{array}$ & $\begin{array}{c}\text { Cout } \\
\text { pour } \\
\text { l'ensembl } \\
\text { e de } \\
\text { l'Etat }\end{array}$ \\
\hline Garde à domicile & 25488 & $48 \%$ & $26 \%$ & $26 \%$ & $:$ & 13253.76 \\
\hline $\begin{array}{c}\text { Garde partagée entre plusieurs } \\
\text { familles }\end{array}$ & 12996 & $35 \%$ & $35 \%$ & $30 \%$ & $:$ & 8447.4 \\
\hline EAJE & 10000 & $10 \%$ & $10 \%$ & $50 \%$ & $30 \%$ & 9000 \\
\hline Assistante maternelle & 7518 & $10 \%$ & $8 \%$ & $82 \%$ & $:$ & 6766.2 \\
\hline Ecole maternelle & 4660 & $6 \%$ & $50 \%$ & & $44 \%$ & 4380.4 \\
\hline Garde par parent CLCA & 4359 & $:$ & $:$ & $:$ & $:$ & 4359 \\
\hline
\end{tabular}

1) Etablissement d'Accueil pour Jeunes Enfants.

Source : Mission de révision générale des politiques publiques relative à la politique familiale, cité par Tabarot (2008), p. 52.

Dans ce contexte, le «coût» budgétaire d'une réforme de l'accueil de la petite enfance peut être estimé en deux étapes, en considérant d'abord les dépenses relatives au congé parental, puis celles induite par l'expansion de l'offre de services d'accueil.

\subsubsection{Un accroissement sensible des dépenses en matière de congé}

On envisage désormais des scénarios où la durée du congé varie, et dont la rémunération est fonction du salaire perçu. Une mesure rigoureuse du coût nécessiterait que soit estimé le taux de recours au congé par niveau de salaire. En l'absence de cette information, nous prenons la situation du Danemark comme étalon puisque les parents d'un nouveau-né ont droit dans ce pays à un congé d'une cinquantaine de semaines de congé total (32 semaines de congé parental qui s'ajoutent aux 18 
semaines de congé maternité), rémunérées à $100 \%$ du salaire en deçà d'un certain plafond - ce plafond étant néanmoins assez bas ${ }^{22}$.

Le coût d'un dispositif similaire appliqué au cas français est alors estimé ici par analogie au cas danois, en supposant que la résultante de ce dispositif impliquera un coût unitaire par enfant comparable au coût danois. Ce coût unitaire dépend bien entendu en toute rigueur du taux de recours au congé et de la distribution des salaires qui varie d'un pays à l'autre. Bien que nous ne puissions tenir compte ici des différences de distribution, on pondère néanmoins le coût unitaire par le différentiel de niveaux de salaires moyens existant la France et le Danemark (encadré 2). Ce nouveau coût représente une hausse unitaire de 4,963€ par enfant par rapport au dispositif actuel pour lequel le coût unitaire est de 7,769€.

Le tableau 3 ( $1^{\text {ère }}$ ligne) applique alors ce nouveau coût unitaire à l'ensemble des naissances annuelles pour estimer le montant de dépenses totales supplémentaires induites au titre du congé parental. Au total, le «nouveau » congé impliquerait une dépense de plus de 10 Milliards d'Euros, contre une dépense actuelle d'un peu plus de 6 Milliards. D'un niveau initial équivalent à $0.37 \%$ du PIB, la dépense effectuée au titre du congé représenterait alors environ $0.60 \%$ du PIB (en supposant ce dernier est stable au niveau de 2005 et que le congé est pris à l'occasion de toutes les naissances).

Tableau 3 : Impact budgétaire d'une réforme de la prise en charge des enfants de moins de trois ans

\begin{tabular}{|c|c|c|c|c|c|c|}
\hline & Coût actuel & $\begin{array}{c}\text { en } \% \text { du } \\
P I B\end{array}$ & $\begin{array}{c}\text { Coût après } \\
\text { réforme }\end{array}$ & $\begin{array}{c}\text { en \% du } \\
P I B\end{array}$ & $\begin{array}{c}\text { Coût « net » de la } \\
\text { réforme }\end{array}$ & $\begin{array}{c}\text { en } \% d u \\
P I B\end{array}$ \\
\hline Dépenses relatives aux congés & $\begin{array}{c}6355271 \\
198\end{array}$ & 0,37 & 10415234000 & 0,60 & 4059962802 & 0.24 \\
\hline $\begin{array}{c}\text { Services d'accueil des enfants de } \\
\text { moins de trois ans }\end{array}$ & $\begin{array}{c}6127541 \\
400\end{array}$ & 0,36 & 9149722452 & 0,53 & 3022181052 & 0.18 \\
\hline Total & $\begin{array}{c}12482812 \\
598\end{array}$ & 0,72 & 19564956453 & 1,13 & 7082143855 & 0.41 \\
\hline
\end{tabular}

\section{Encadré 2 : Estimation du coût du congé parental}

Le coût du congé parental est estimé à partir des dépenses réalisées dans chaque pays au titre des congés maternité, paternité et parental rapporté à chaque naissance. Ces dépenses égalent €7,769 par enfant en France en 2005 et $€ 18,155$ au Danemark.

Pour obtenir une estimation du coût réprésenté par la transposition du dispositif danois au cas français, on tient compte ici de la différence de niveau de salaire moyen existant entre ces deux pays (€30,156 en France, et 42,999 au Danemark). Le coût par enfant du dispositif de congé à la danoise appliqué au cas français est alors estimé à 12,733 € pour un an en 2005, obtenu par le produit du coût unitaire danois $(18,155 €)$ pondéré par le rapport des salaires moyens de ces deux pays. Les dépenses totales en matière de congé après réforme (en supposant que la fécondité ne soit pas affectée) s'élèvent donc à 10,415 milliards si celui-ci est fixé à un an, ce qui correspond à $0.6 \%$ du PIB.

Pour obtenir le coût total du nouveau format de congé, on multiplie le coût unitaire par le nombre de naissances annuelles, 817,987 obtenu ici comme moyenne du nombre de naissances enregistrées de 2005 à 2007, en supposant que le nombre de naissances sera relativement stable dans les années futures.

\subsubsection{L'expansion des services de garde : un coût variable selon le mode...}

L'impact budgétaire de l'expansion de l'offre de services dépend, quant à lui, du nombre de places devant être créées pour répondre aux besoins induits par un congé plus court. Il dépend aussi du mode d'accueil privilégié pour accroître les capacités d'accueil. L'estimation du coût budgétaire de cet accroissement de l'offre dépend bien entendu du point de départ et de l'objectif fixé. Or ces deux points de référence font l'objet d'estimations variables. Le point de départ, tout d'abord, doit considérer non pas le taux de fréquentation effectif des services d'accueil, mais leur capacité d'accueil

${ }^{22}$ En fait, les parents en congé parental perçoivent un revenu de remplacement égal à leur salaire en deçà jusqu'à un maximum d'environ $€ 100$ par jour. Ce plafond est donc relativement bas, ce qui aboutit à des taux de remplacement très fortement décroissant avec le niveau de salaire, dès que celui-ci est égal ou supérieur au salaire moyen (voir OCDE 2011, Base de données sur la Famille, indicateur PF2.4). 
«théorique », qui tient compte du fait que certaines places ne sont pas utilisées, ou sous-utilisées car elles pourraient être partagées par plusieurs enfants accueillis en relais. L'Observatoire National de la Petite Enfance publie un taux de capacité d'accueil théorique qui comptabilise des places d'accueil existantes et non le nombre d'enfant accueillis. Cette capacité est calculée pour les enfants de moins de trois ans en tenant comptes de places mises à disposition par les assistants maternels (qui peuvent également accueillir des enfants plus âgés), les établissements d'accueil collectif ou familial, les salariés à domicile et l'école maternelle. Les informations sur l'offre n'étant pas toutes disponibles, la capacité d'accueil théorique est parfois approchée par des données sur l'accueil constaté (voir les détails dans ONPE, 2009). Le potentiel d'accueil est alors estimé à niveau théorique de 44,3\% des enfants - ce qui est en réalité très peu différent du taux de fréquentation réelle donné par l'enquête EU-SILC (tableau 1).

L'objectif d'accueil à atteindre peut, quant à lui, être raisonnablement fixé aux deux tiers des enfants de moins de trois ans qui correspond aux deux tiers de la période pendant laquelle les enfants de moins de trois ans devront être accueillis dans un service si les parents prennent en charge l'enfant dans sa première année ${ }^{23}$. Certains parents peuvent toutefois vouloir confier leur enfant à un service formel avant l'âge de 1 an, comme c'est le cas actuellement. D'autres au contraire continueront à davantage mobiliser des réseaux informels. L'objectif des deux tiers est donc à concevoir comme un seuil autour duquel la demande est amenée à fluctuer (selon des scenarios que nous évoquerons ciaprès).

Le tableau 3 ( $2^{\text {ème }}$ ligne) estime alors l'impact budgétaire d'une expansion de l'offre d'accueil permettant de passer de la capacité théorique actuelle (44\%) aux $66 \%$ des enfants de moins de trois ans fixés comme objectif $(534,963$ enfants de moins de trois ans $(22 \%)$ seront ainsi couverts en plus par cette expansion). Le coût de l'expansion des services d'accueil est en outre obtenu en appliquant au nouveau taux de couverture le coût unitaire estimé pour 2005 (Encadré 3). Les dépenses d'accueil des enfants de moins de 3 ans passent alors d'un montant égal à $0.36 \%$ du PIB à une dépense théorique équivalente à $0.53 \%$ de la valeur du PIB de 2005.

\section{Encadré 3 : Estimation du coût de l'augmentation de l'offre d'accueil}

Le coût estimé de l'augmentation de l'offre de garde pour les enfants de moins de trois ans est obtenu par une règle de trois appliquée aux dépenses «actuelles » réalisées en matière de mode d'accueil telles qu'elles sont enregistrée dans la base de données sur les dépenses sociales de l'OCDE, en supposant que les deux tiers des enfants seront couverts par un mode d'accueil. Cette règle suppose implicitement que la structure des coûts de l'accueil demeure constante avec l'augmentation des capacités d'accueil. Les taux de recours aux différents modes d'accueil sont empruntés à l'enquête mode de garde 2007, où $26 \%$ des enfants de moins de trois ans sont pris en charge par une assistante maternelle de façon principale ou secondaire ; $18 \%$ sont accueillis en crèche ; $3 \%$ sont gardés à domicile ; $6 \%$ sont accueillis en milieu scolaire.

Les scénarios envisageant différentes alternatives de mode d'accueil tiennent compte des différences de taux d'occupation des places disponibles pour l'accueil. Le nombre de places nécessaires à l'accueil des 534,963 supplémentaires dépend ainsi du nombre d'enfants que peut accueillir chaque place, ce ratio variant selon le mode d'accueil (1.33-1.5 pour les établissements collectifs ; 0.86 pour les assistantes maternelles). Le coût supplémentaire d'un accueil en établissement collectif varie alors selon le nombre de places jugées nécessaire selon l'hypothèse retenue pour le taux de remplissage. Ce nombre de places varie de 402,089 si un taux d'occupation de 1,33 par place est appliqué à 534,963 si chaque enfant est supposé occuper une place entière. Le coût de fonctionnement induit par l'offre supplémentaire varie alors de 3,2 à 3,6 milliards d'euros. S'ajoute à ce coût des dépenses d'investissement 7,8 et 8,8 milliards selon l'hypothèse faite sur le ratio d'enfants accueillis par place (1,33 ou 1.5). Ces dépenses ne sont pas incluses dans les dépenses annuelles puisqu'elles sont amorties sur plusieurs années. Le même raisonnement est appliqué pour l'accueil par des assistantes maternelles sans inclure dans ce cas de coûts d'investissement.

Le mode d'accueil privilégié pour accroître l'offre a aussi une incidence sur le coût. Un ordre de grandeur des variations possibles peut être estimé, à condition d'estimer le nombre de places devant être créées pour chaque mode de garde. Ce nombre de places varie car le nombre d'enfants couverts par chaque place diffère selon le mode.

\footnotetext{
${ }^{23}$ A titre de comparaison, on peut rappeler qu'au Danemark où la somme des congés maternel et parental n'est pas supérieure à 50 semaines, près de $85 \%$ des enfants sont en centre d'accueil dès leur deuxième année, alors qu'ils ne sont que $15 \%$ dans leur première année, pour l'année 2005.
} 
Considérons d'abord le nombre de places en crèches nécessaires pour accueillir les 534,963 enfants supplémentaires devant être accueillis. Il faut, pour cela, tenir compte du taux d'occupation des places d'accueil qui peuvent servir à plusieurs enfants si la prise en charge est effectuée à temps partiel ou de façon alternée. Pour le cas des établissements collectifs, on estime qu'une place en crèche permet d'accueil une moyenne de 1,33 enfants $^{24}$. Le nombre de places nécessaires pour répondre à la « demande » supplémentaire oscille donc entre 402,089 (en tenant compte du taux d'occupation ${ }^{25}$ ) et 534,963 si chaque enfant occupe une place entière. Les coûts correspondants de l'accroissement de l'offre sont alors compris entre 0.19 et $0.21 \%$ du PIB. Au total, le coût annuel d'une prise en charge de tous les enfants de moins de six ans varie de 0.33 à $0.6 \%$ du PIB selon la séquence choisie entre l'accueil en établissement collectif suivi par l'entrée en école maternelle. Ce coût correspond aux dépenses de fonctionnement mais néglige l'investissement initial nécessaire à la création de nouveaux établissements et qui peut être estimé entre 0.45 et $0.51 \%$ du PIB.

Le même raisonnement peut être tenu pour le cas où la demande d'accueil est satisfaite par un accroissement de l'effectif d'assistantes maternelles. Le taux d'occupation est ici très différent du cas précédent puisqu'on observe une sous-utilisation du potentiel offert par ces dernières : une place disponible permet en effet de couvrir en moyenne 0.86 enfants $^{26}$. Si ce ratio est maintenu, le nombre de places nécessaires pour couvrir les 534,963 demandes supplémentaires sera alors de 620,462 places. Le coût correspondant est alors égal à $0.24 \%$ du PIB, mais pourrait être ramené à $0.21 \%$ si le ratio entre places et enfants couverts est ramené à 1 . Autrement dit, on n'observe pas de différence très significative en termes d'impact budgétaire entre les deux options considérées ici: le développement de l'accueil en établissement collectif ou la prise en charge par des assistantes maternelles.

\subsection{3... Et selon le «parcours de garde » durant la petite enfance}

Le coût financier est, en revanche, plus sensible à la séquence des modes de garde qui se succède au cours de l'enfance. Le mode de garde suivant la période de congé importe alors dans ce cas. Pour estimer ces différences, on évalue le coût de la prise en charge d'un enfant au cours de ses six premières années, en faisant référence aux six parcours présentés au début de la section 3 (Tableau 4). Ces six scénarios sont évalués en tenant compte de la possibilité d'être accueilli en établissement collectif (cas A), ou d'être pris en charge par une assistante maternelle (cas B).

La comparaison de tous les scénarios montre que, globalement, ce sont les options qui combinent la prise en charge réalisée par une assistante maternelle, et un accueil en école maternelle précoce qui ont un moindre poids budgétaire. Le scénario le moins onéreux pour l'État est celui qui voit s'enchaîner une année de congé parental, une année de prise en charge par une assistante maternelle, et une entrée en école maternelle s'effectuant à 2 ans. L'accueil généralisé en crèches dès l'âge de 1 ans jusqu'à 3 ans est quant à lui le scenario le plus coûteux. Enfin, le scenario qui viserait à augmenter la durée du congé parental au-delà d'une année serait plus coûteux, quel que soit le mode d'accueil lui succédant.

\footnotetext{
${ }^{24}$ Ce chiffre est obtenu par le ratio entre les 441,712 enfants accueillis en établissement collectif et les 332,000 places recensés en 2007 (Ananian et Robert-Bobée, 2009).

${ }^{25}$ Chiffre obtenu en divisant les 534963 demandes supplémentaires par le taux d'occupation de 1,33.

${ }^{26}$ Les assistantes maternelles couvrent aujourd'hui $26 \%$ des enfants, qui leur sont confiés en mode de garde principal ou secondaire, alors que le nombre de places disponibles est évalué à 710,000. Le décalage entre la localisation de l'offre et celle de la demande doit permettre d'expliquer en partie cette situation.
} 
Tableau 4 : Un coût variable de la prise en charge variable selon le parcours

Cas A : congé suivi d'un accueil en établissement collectif

\begin{tabular}{|c|c|c|c|c|c|c|c|c|c|c|c|c|c|c|c|}
\hline & \multicolumn{3}{|c|}{ Congé } & \multicolumn{5}{|c|}{ Accueil dans un Etablissement collectif } & \multicolumn{3}{|c|}{ École maternelle } & \multirow{2}{*}{$\begin{array}{l}\text { Total } \\
\text { par } \\
\text { enfant }\end{array}$} & \multirow{2}{*}{$\begin{array}{c}\text { Coût } \\
\text { annuel } \\
\text { par } \\
\text { enfant }\end{array}$} & \multirow{2}{*}{$\begin{array}{c}\text { Total } \\
\text { annuel } \\
\text { pour tous } \\
\text { les } \\
\text { enfants } \\
\text { (en \% du } \\
\text { PIB } \\
2005 \text { ) } \\
\end{array}$} & \multirow{2}{*}{$\begin{array}{c}\text { Total de } \\
\text { dépenses pour } \\
\text { une } \\
\text { génération sur } \\
6 \text { ans (en \% } \\
\text { du PIB de } \\
2005 \text { ) }\end{array}$} \\
\hline & $\begin{array}{c}6 \\
\text { mois }\end{array}$ & $\begin{array}{l}12 \\
\text { mois }\end{array}$ & $\begin{array}{c}18 \\
\text { mois }\end{array}$ & $\begin{array}{c}12 \\
\text { mois }\end{array}$ & $\begin{array}{c}18 \\
\text { mois }\end{array}$ & $\begin{array}{c}24 \\
\text { mois }\end{array}$ & $\begin{array}{c}30 \\
\text { mois }\end{array}$ & $\begin{array}{c}36 \\
\text { mois }\end{array}$ & $\begin{array}{c}30 \\
\text { mois }\end{array}$ & $\begin{array}{c}36 \\
\text { mois }\end{array}$ & $\begin{array}{c}42 \\
\text { mois }\end{array}$ & & & & \\
\hline $\begin{array}{c}\text { Scenario } \\
\mathrm{a}\end{array}$ & & 12,733 & & & & 18,000 & & & & 13,141 & & 43,874 & 7,312 & 0.35 & 2.06 \\
\hline $\begin{array}{c}\text { Scenario } \\
\mathrm{b}\end{array}$ & & 12,733 & & & & & 22,500 & & 10,961 & & & 46,184 & 7,697 & 0.36 & 2.17 \\
\hline $\begin{array}{c}\text { Scenario } \\
\mathrm{c}\end{array}$ & 6,366 & & & & & & 22,500 & & & 13,141 & & 42,008 & 7,001 & 0.33 & 1.99 \\
\hline $\begin{array}{c}\text { Scenario } \\
\mathrm{d}\end{array}$ & 6,366 & & & & & & & 27,000 & 10,961 & & & 44,317 & 7,386 & 0.35 & 2.10 \\
\hline $\begin{array}{c}\text { Scenario } \\
\mathrm{e}\end{array}$ & & & 19,099 & & 13,500 & & & & & 13,141 & & 45,740 & 7,623 & 0.36 & 2.17 \\
\hline $\begin{array}{c}\text { Scenario } \\
\mathrm{f}\end{array}$ & & & 19,099 & 9,000 & & & & & & & 15,331 & 43,431 & 7,238 & 0.34 & 2.06 \\
\hline
\end{tabular}

Cas B : congé suivi d'une prise en charge par une assistante maternelle

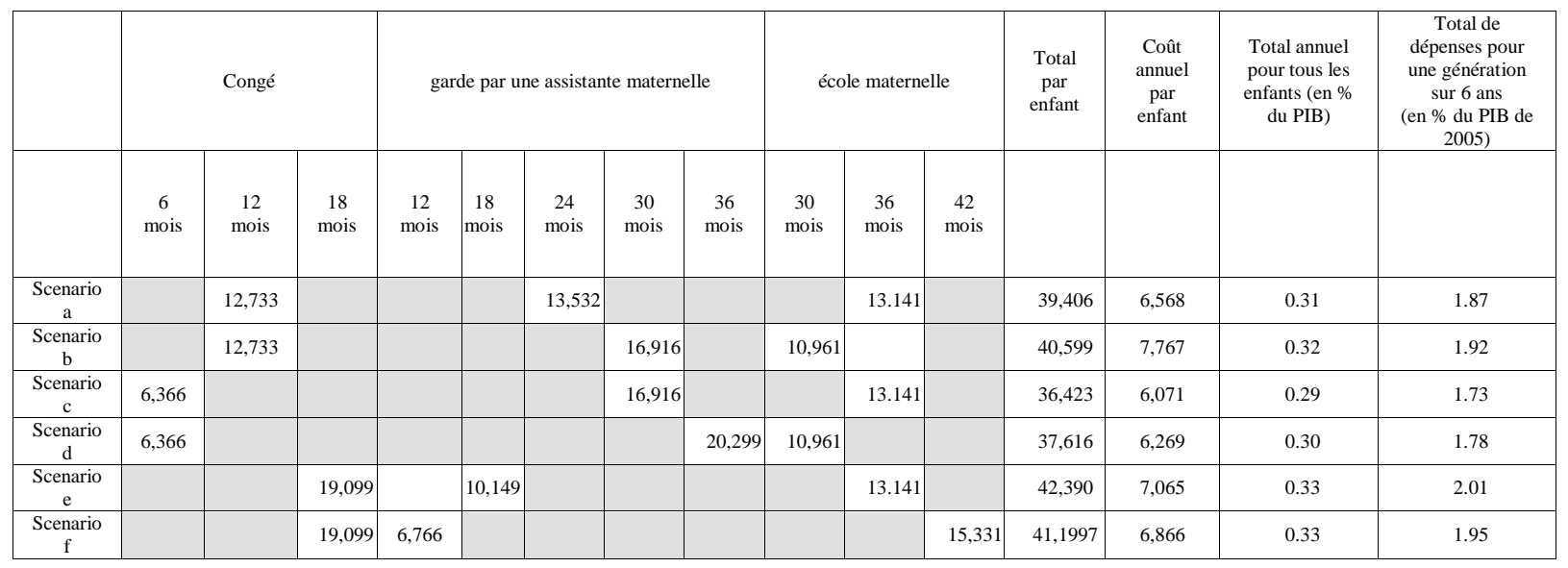

Investir dans l'accueil de la petite enfance pour améliorer les capacités de choix des familles

L'investissement massif réalisé par l'Etat en direction des familles avec enfants en France est l'une des clés de voûte du système qui permet à ce pays d'afficher des performances en matière de fécondité, d'emploi des femmes aux âges de la maternité ou de pauvreté infantile comparativement meilleures que la moyenne des pays de l'OCDE. L'une des caractéristiques de ces aides est leur caractère très diversifié, au sens où les familles bénéficient de prestations financières diverses et d'aides en matières de services d'accueil à destination des très jeunes enfants plus développées que dans beaucoup d'autres pays, à l'image des pays nordiques. Il n'en reste pas moins certaines spécificités ancrées sur l'affichage d'une politique de « libre choix » qui vise à permettre aux parents de choisir le mode de prise en charge de leur enfant en bas-âge. Cette liberté est néanmoins fortement circonscrite par les contraintes financières et professionnelles qui orientent les choix réalisés en matière de prise en charge des enfants et de la façon de concilier celle-ci à une activité professionnelle. Ces contraintes ont, en outre, des conséquences variables pour les hommes et les femmes (et pour les femmes entre elles) puisque ce sont la plupart du temps les mères qui prennent un congé de plusieurs mois ou année pour s'occuper d'un enfant en bas-âge, et qui en subissent des pénalités en terme d'employabilité ou de trajectoires professionnelles qui ne s'estompent pas toujours dans le temps. 
Les opportunités de concilier ou non travail et vie familiale continuent d'être assez variables avec le niveau de vie des ménages et les contraintes professionnelles auxquelles les parents peuvent s'ajuster avec plus ou moins de marge. La diversité des aides en matière d'accueil est importante pour répondre à des situations diverses, mais leur dualisme a pour effet d'accroître certaines inégalités sur le marché du travail qui préexistent à la naissance d'enfants : d'un côté, le recours à un mode de garde individuel est largement encouragé pour les ménages dont le revenu est relativement élevé et qui bénéficient pour cela d'aides qui réduisent fortement la charge financière qu'ils ont alors à supporter. L'effort financier qui incombe aux ménages plus modestes pour bénéficier de ce mode de garde demeure sensiblement plus élevé. En l'absence de place en crèches, les mères disposant de revenus limités sont alors fortement encouragées à interrompre leur activité en bénéficiant du CLCA ; le retour à l'emploi ne se fait alors pas toujours. Aussi, bien que ce soit involontaire, les deux régimes d'aides bénéficient de façon contrastée aux deux extrêmes de l'échelle des revenus.

Dans ce contexte, un accroissement de l'offre de services d'accueil accompagnée d'un congé mieux rémunéré qu'aujourd'hui pourrait permettre d'accroître les capacités de choix des familles ${ }^{27}$. Un congé rémunéré à proportion du salaire et plus court aurait ainsi l'avantage d'être une option plus attractive pour les femmes et les hommes dont le salaire est nettement supérieur à l'actuelle allocation de libre choix d'activité. Un revenu plus élevé durant la période de congé est aussi à même de garantir un revenu plus stable et une meilleure protection face au risque pauvreté - même si la période de congé est plus courte pour tenir compte des contraintes budgétaires. Un congé plus court, s'il est accompagné d'un accroissement de l'offre d'accueil suffisant peut aussi faciliter le retour à l'emploi des femmes désireuses de poursuivre une activité ou une carrière, et avoir pour effet de limiter les formes de discrimination pouvant être associée à un départ en congé. Enfin, un tel congé semble aussi un pré requis favorable à un partage plus égal de la prise en charge des enfants, et donc à un partage plus équilibré entre hommes et femmes des risques liés à une interruption d'emploi suivant la naissance d'enfants.

Une telle réforme de l'accueil de la petite enfance a un coût financier puisque, en plus des dépenses induites en matière de congé (environs $0.25 \%$ du PIB), une augmentation de l'offre d'accueil d'au moins 400,000 places en crèche paraît nécessaire accueillir l'ensemble des enfants de moins de trois ans à l'expiration d'une année de prise en charge par les parents ${ }^{28}$. Le coût «brut » de cette expansion de l'offre d'accueil de la toute petite enfance oscille entre 0.20 et $0.25 \%$ selon le scénario retenu. Au total, c'est donc une hausse des dépenses équivalent à $0.5 \%$ de PIB qui seraient requise pour l'accueil des enfants de moins de trois ans, passant d'environ $0.70 \%$ du PIB aujourd'hui à $1.20 \%$ (et $1.80 \%$ lorsqu'on étend l'estimation à tous les enfants de moins de 6 ans). Le coût peut varier en fonction du mode privilégié pour accroître les capacités d'accueil, une augmentation de l'accueil par des assistant(e)s étant la moins coûteuse pour le budget de l'Etat si les règles de subvention et les taux d'accueil demeurent identiques aux taux actuels. En revanche, l'augmentation du nombre de place en crèche ou établissement collectif est l'option qui bénéficierait le plus aux familles à revenus modestes puisque le coût pour les familles est plus faible.

L'estimation que nous avons faite ici a néanmoins ses limites. Tout d'abord, les bénéfices qui peuvent être directement induit par l'investissement ainsi réalisé ne sont pas pris en compte. Or, des bénéfices financiers peuvent être générés rapidement par les recettes fiscales induites si le taux d'emploi des mères ou leur volume de travail sont accrus en raison de l'augmentation de l'offre d'accueil des enfants. Ces bénéfices peuvent être substantiels puisqu'on peut estimer à 160,000 le nombre de

\footnotetext{
${ }^{27}$ Un congé de ce type pourrait se substituer ou s'ajouter au dispositif actuel. Nous ne discutons pas ici ce point. Le maintien d'une option de congé long continuerait probablement d'être privilégié par les ménages avec de faibles revenus et dont les conditions de travail sont particulièrement difficiles à concilier avec l'activité des deux parents. Les femmes avec de basse qualifications et dont l'emploi évolue peu avec l'ancienneté peuvent en particulier préférer cette option à un conge court.

${ }^{28}$ L'augmentation envisagée ici est bien supérieure à celle du Plan Petite Enfance adopté en 2006 qui prévoyait la création d'environ 60,000 places en crèches sur 5 ans, à ajouter aux 160,000 enfants accueillis par 60,000 assistantes maternelles en plus.
} 
femmes pouvant entrer en emploi si le taux d'emploi des mères s'ajuste progressivement à celui des femmes de 15 à 64 ans sans enfant. En supposant une productivité par travailleur stable, la production additionnelle réalisée par ce contingent équivaut à $0.6 \%$ PIB. Le supplément de richesses induites pourrait donc être d'un montant au moins équivalent aux dépenses initialement consenties - en supposant évidemment l'absence de rationnement sur le marché du travail et aucun effet d'éviction entre hommes et femmes.

La schématisation très simple que nous avons adoptée a aussi d'autres limites, puisqu'elle n'est pas fondée sur une estimation des réponses comportementales que pourraient avoir les hommes et les femmes face à une modification des conditions d'accueil des enfants. Une estimation des taux de recours et des réactions de l'offre de travail des hommes et des femmes permettrait bien-sûr de préciser les scenarios, et d'examiner quels ménages pourraient bénéficier plus particulièrement des différents scénarios. Une simulation «dynamique» des trajectoires professionnelles permettrait, en outre, de mieux fonder des scénarios sur les bénéfices et l'évolution des inégalités de long terme. Enfin, la qualité des modes d'accueil n'est pas directement considérée ici, alors qu'elle est un paramètre déterminant des bénéfices éventuels que peuvent tirer les enfants des services d'accueil. Bien que la littérature suggère l'existence de tels bénéfices, une évaluation plus précise de ceux-ci permettrait de fonder un consensus plus large sur l'opportunité d'une réforme. 


\section{Références}

Adema W., Thévenon O. (2008), «Les politiques de conciliation du travail et de la vie familiale en France au regard des autres pays de l'OCDE », Recherches et Prévisions, 933, pp. 51-72.

Ananian S., Robert-Bobée I. (2009), « Modes de garde et d'accueil des enfants de moins de 6 ans en $2007 »$ Etudes et Résultats, DREES, 678.

Baker, M., Gruber, J. \& Milligan, K. (2005). Universal child care, maternal labour supply and family well-being. Toronto: University of Toronto. Department of Economics.

Baldi S., Chapple S. (2011), "Birth-related Leave Durations across the OECD, 1969-2008", Social, Employment and Migration Working Paper, OECD Publishing, Paris, forthcoming.

Bechtel J., Caussat L., Courtioux P., Laib N., Le Minez S. et Mirouse B. (2005), La politique familiale française : coûts et bénéficiaires, Complément J, in Godet M. et Sullerot E., in La Famille, une affaire publique, CAE, rapport $n^{\circ} 57$, Paris, La Documentation française.

Baker M., Gruber J. et Milligan K., 2005, « Universal childcare, maternal labour supply and family well-being », Toronto: University of Toronto, Department of Economics.

Baum Ch., 2003, "Does early maternal employment harm child development? An analysis of the potential benefits of leave taking", Journal of Labor Economics, 21(2):409-448.

Belsky J., 2004, «La quantité de temps de garde et le développement socio-émotionnel du jeune enfant », Recherche Clinique, 16(1):5-15.

Bernal R. et Keane M., 2006, « Child care choices and children's cognitive achievement: The case of single mothers », Working Paper, Northwestern University.

Berger E. (2008), «Les prestations familiales et de logement en 2007. Une redistribution en faveur des familles nombreuses et des personnes isolées », Etudes et Résultats, 674, décembre.

Blasko Z. (2008), "Does early maternal employment affect non-cognitive children outcomes? - A literature review", Budapest Working Papers on the Labour Market, 5.

Boyer D. (2004), «Les pères bénéficiaires de l'allocation parentale d'éducation : révélateurs de nouvelles pratiques paternelles? », Recherches et Prévisions, $\mathrm{n}^{\circ}$ 76:53-62.

Bressé S. et Galtier B. (2006), « La conciliation entre vie professionnelle et vie familiale selon le niveau de vie des familles », Études et Résultats, $n^{\circ} 465$.

Breton D., Prioux F. (2009), "The one-child family: France in the European context", Demographic Research, 20: 657-692.

Brin H. (2005), Enjeux démographiques et accompagnement du désir d'enfants des familles, Conférence de la Famille 2005.

Brooks-Gunn J., Han W., et Waldfogel J., 2002, "Maternal employment and child cognitive outcomes in the first three years of life: The NICHD study of early child", Child Development, 73(4):10521072.

Caillé J.-P. (2003), «Faut-il développer la scolarisation à deux ans ?», Éducation et formations, n 66, pp.7-11.

Centre d'Analyse Stratégique (CAE) (2007), Rapport sur le Service Public de la Petite Enfance, http://www.strategie.gouv.fr/IMG/pdf/Rapport_SPPE_VF14022007-2.pdf

Cour des Comptes (2008), La Sécurité Sociale, Rapport annuel.

Clergeau M.F. (2009), Rapport d'information sur la Prestation d'Accueil du Jeune Enfant, Assemblée Nationale, MECSS.

Cohen S., (1995), De la crèche à l'école. Paris : Nathan Pédagogie, 1995. 
Crenner E. (2011), «Prendre un congé parental total : une décision qui dépend essentiellement du nombre d'enfants et de l'emploi occupé auparavant », Etudes et Résultats, 751, DREES.

Currie J. (2001), "Early Childhood Education Programs", Journal of Economic Perspectives, 15(2): 213-238.

Datta-Gupta N., Simonsen M. (2010), "Non cognitive children outcomes and universal high quality child care", Journal of Public Economics, 94(1-2), pp. 30-43.

DeCicca Ph., Smith J. (2011), "The long-run impacts of early childhood education evidence from a failed policy experiment", National Bureau of Economic Research (NBER), Working Paper 17085.

Duvander, A.-Z., Lappegard, T., \& Andersson, G. (2010). Family policy and fertility: A comparative study on the impact of fathers' and mothers' use of parental leave on continued childbearing in Norway and Sweden. Journal of European Social Policy, 20(1), 45-57.

Duvander, A.-Z. et M. Johansson (2010), "How Effective Are Reforms Promoting Fathers" Parental Leave Use?”, European Population Conference, Vienna, 1-4 September.

Esping-Andersen G. (2008), Trois leçons sur l'Etat Providence, Seuil, coll. La république des Idées, Paris.

Eversston, M. and A.-Z. Duvander (2010), "Parental Leave - Possibility or Trap? Does Family Leave Length Effect Swedish Women's Labour Market Opportunities?", European Sociological Review, Advance Access published, DOI:10.1093/esr/jcq018.

Eurobarometer (2008), Family life and the needs of an ageing population, 247, Bruxelles.

Eydal, G.B. and I.V. Gislason (2008), "Equal Rights to Earn and Care - Paid Parental Leave in Iceland", Felags og Tryggingamala Raduneytid.

Eydoux A. et Letablier M.-T. (2007) «Les familles monoparentales », Document de recherche $n^{\circ} 36$, CEE.

Eydoux A., Letablier M.-T. et Sylla Samba (2006), La conciliation vie professionnelle et vie familiale des familles pauvres ou précaires, in Les travaux de l'Observatoire 2005-2006, Observatoire national de la pauvreté et de l'exclusion sociale, Paris, La Documentation française :161-198.

Godefroy P., Missègue N., Pujol J., Tomasini M. (2011), "Inégalités de niveaux de vie et pauvreté", INSEE.

Goux D., Maurin E. (2009), "Preschool enrolment, Mothers participation in the labour market and children's subsequent outcomes", PSE working paper.

Gregg P, Harkness S. et Machin S., 1999, Child development and family income, York: Joseph Rowntree Foundation.

Gregg P., Washbrook E., Propper C., Burgess S. (2005), "The Effects of a Mother's Return to Work Decision on Child Development in the UK", Economic Journal, 115 (501), pp. F48 - F80.

Han W., Waldfogel J. et Brooks-Gunn J., 2001, "The effects of maternal employment on later cognitive and behavioral outcomes", Journal of Marriage and the Family, 63:336-54.

Haut Conseil à la Famille (2011), Avis sur l'architecture des aides aux familles : quelles évolutions pour les 15 prochaines années ?, Note du 28 avril 2011.

Heckman J., Masterov (2007), « The productivity argument for investing in young children », NBER Working Paper series, $n^{\circ} 13016$, National Bureau of Economic Research.

Huerta M., Adema W., Baxter J., Corak M., Deding M., Gray M.C., Han W.J. and J. Waldfogel (2011), "Early Maternal Employment and Child Development in Five OECD Countries", Social, Employment and Migration Working Papers, OECD Paris. 
IGAS (2011), L'égal accès des femmes et des hommes aux responsabilités professionnelles et familiales dans le monde du travail, Rapport prepare par B. Grésy et Ph. Dole.

Kamerman S., Neuman M.,Waldfogel J. et Brooks-Gunn J., 2003, « Social policies, family types and child outcomes in selected OECD countries ». Paris, France: OECD working paper.

Kesteman N. (2009), «Opinions des Français sur les politiques familiales et sociales début 2009 », essentiel 92, CNAF.

Lefebvre P., Merrigan Ph., Roy-Desrosiers F. (2011), "Quebec's Childcare Universal Low Fees Policy 10 Years After: Effects, Costs and Benefits", Working paper 11-01. Centre Interuniversitaire sur le Risque, les Politiques Economiques et l'Emploi (CIRPEE), Montréal.

Legendre E., Vanovermeir S. (2011), «Situations professionnelles à l'entrée et à la sortie du complément de libre choix d'activité (CLCA) », Etudes et Résultats, 750, DREES.

Letablier M.-Th., Thévenon O. (2009), "The effectiveness of policies on families and children's wellbeing", in Letablier M.Th., Luci A., Math A., Thévenon O, The costs of raising children and the effectiveness of supporting parenthood policies in European countries: a Literature review, Rapport pour la Commission Européenne, INED.

Jaumotte F. (2003), « Female labor force participation: past trends and main determinants in OECD countries », OECD Economic Department Working Paper, 375.

Méda D., Périvier H. (2007), Le deuxième âge de l'émancipation, La République des Idées, Le Seuil.

Meurs, D., A. Pailhé and S. Ponthieux (2011), "Child-related Career Interruptions and the Gender Wage Gap in France", Annales d'Economie et de Statistique, forthcoming.

Miranda V. (2011), "Cooking, Cleaning and Volunteering: Unpaid Work around the World", Social, Employment and Migration Working Papers, OECD Paris.

Observatoire National de la Petite Enfance (2008), L'accueil de la Petite Enfance en 2007, CNAF.

OCDE (2011), Assurer le bien-être des familles, Paris, OCDE Publications.

OCDE (2009), Assurer le bien-être des enfants, Paris, OCDE Publications.

OCDE (2007), Bébés et employeurs - Comment réconcilier travail et vie de famille, une synthèse des résultats, Paris, OCDE Publications.

Office Statistique Federal (2010), "Geburten und Sterbefaelle", online database: www.destatis.de, accessed 26 September 2010.

Pailhé A., Solaz A. (2007), «What makes French employees so happy with their balance between family and Work? The impact of firm's family-Friendly Policies", IZA / Bertelsmann Foundation Workshop: Blancing Work and Family Life: Comparative Assessment and Institutional Reform, IZA, Bonn, November 23-24.

Piketty Th. (2005), «L'impact de l'allocation parentale d'éducation sur l'activité féminine et la fécondité en France, 1982-2002 », in Histoires de familles, histoires familiales, Les cahiers de l'INED. $\mathrm{N}^{\circ} 156$, p. 79-109.

Ruhm C. (2003), « How well do parents with young children combine work and family life », NBER Working paper, $\mathrm{n}^{\circ}$ 10247, National Bureau of Economic Research, Cambridge, MA.

Ruhm C. (2004) "Parental employment and child cognitive development", Journal of Human Resources, 39(1):155-192.

Ruhm C. (2000), "Parental leave and Child Health", Journal of Health Economics, 19(6):931-960.

Stafford F., 1987, Women's work, siblings competition and children's school performance, American Economic Review, 77(5): 972-980. 
Tabarot M. (2008), Rapport sur le développement de l'offre d'accueil de la petite enfance, http://www.premier-ministre.gouv.fr/IMG/pdf/Rapport_Tabarot.pdf

Tanaka S. (2005), «Parental leave and Child Health across OECD countries », Economic Journal, 115(501):F7-F28.

Thévenon, O. (2011), "Family Policies in OECD countries: A Comparative Analysis", Population and Development Review, Vol. 37, No 2, pp. 57-87.

Thévenon O. (2009) «L'augmentation de l'activité des femmes en Europe: progrès de la conciliation ou polarisation des comportements ? » Population 64(2) :263-304.

Thévenon O. (2006) : «Régimes d'État Social et Convention Familiale : une analyse des régulations

Emploi-Famille », Économies et Sociétés : série « socio-économie » du travail, $\mathrm{n}^{\circ} 27$ (6) : 1137-71.

Thévenon O., Gauthier A. (2010), "Variations de la fécondité dans les pays développés: disparités et influences des politiques d'aides aux familles », Politiques Sociales et Familiales, 100 :7-21.

Toulemon L., Pailhé A., Rossier C. (2008), «France: high and stable fertility", Demographic Research, 19(16): 503-556. Special Collection 7: Childbearing Trends and Policies in Europe.

Waldfogel J., Wen-Jui H. et Brooks-Gunn J., 2002. "The effects of early maternal employment on child Cognitive development", Demography, 39(2):369-392.

Whiteford P. et Adema W. (2007), «What works best in reducing child poverty: a benefit or work strategy?», OECD Social, Employment, and Migration, Working Paper 51, OCDE, Paris.

Zhang, X. (2010), "Can Motherhood Earnings Losses Be Ever Regained? Evidence from Canada", Journal of Family Issues, Vol. 31, No. 12, pp. 1671-1688.

Zazzo B., 1984, Un grand passage: de l'école maternelle à l'école élémentaire. Paris : PUF.

Zazzo B., 1997, «L'éducation préscolaire », INRP, Revue française de pédagogie № 119. 